\title{
A multiscale mean-field homogenization method for fiber-reinforced composites with gradient-enhanced damage models
}

\author{
L. Wu $u^{\mathrm{a}, \mathrm{b}, *}$, L. Noels ${ }^{\mathrm{a}}$, L. Adam ${ }^{\mathrm{c}}$, I. Doghri $\mathrm{i}^{\mathrm{c}, \mathrm{d}}$ \\ ${ }^{a}$ University of Liège CM3, B-4000 Liège, Belgium \\ ${ }^{b}$ Northwestern Polytechnical University, 710072 Xi'an, China \\ ${ }^{c}$ e-Xstream Engineering, Rue du Bosquet, 7, 1348 Louvain-la-Neuve, Belgium \\ ${ }^{d}$ Université Catholique de Louvain, iMMC, Bâtiment Euler, Avenue G. Lemaître 4, 1348 \\ Louvain-la-Neuve, Belgium
}

\begin{abstract}
In this work, a gradient-enhanced homogenization procedure is proposed for fiber reinforced materials. In this approach, the fiber is assumed to remain linear elastic while the matrix material is modeled as elasto-plastic coupled with a damage law described by a non-local constitutive model. Toward this end, the mean-field homogenization is based on the knowledge of the macroscopic deformation tensors, internal variables and their gradients, which are applied to a micro-structural representative volume element (RVE). The macro-stress is then obtained from a homogenization procedure. The methodology holds for 2phase composites with moderate fiber volume ratios, and for which, at the RVE size, the matrix can be considered as homogeneous isotropic and the ellipsoidal fibers can be considered as homogeneous transversely isotropic. Under these assumptions, the method is successfully applied to simulate the damage process occurring in unidirectional carbon-fiber reinforced epoxy composites submitted to different loading conditions.
\end{abstract}

Keywords: Finite elements, Gradient-enhanced, Mean-Field Homogenization, Fiber-reinforced materials, Composite

\section{Introduction}

As composite materials are multiscale in nature, from the accuracy aspect of the structural analysis, it would be better to refer directly to the microstructure deformations during numerical simulations. However, as such direct numerical simulations are often too complex to handle and as the computation costs are

\footnotetext{
* Corresponding author, Phone: +324366 94 53, Fax: +32 43669505

Email address: L.Wu@ulg.ac. be (L. Wu )
} 
unaffordable, composite structural analyzes are usually carried out at the macroscopic scale with homogenized material properties, which are derived from the microscopic analysis.

Homogenization techniques are known to be efficient tools to derive those homogenized material properties analytically and/or numerically from the constituents properties and from the microstructure of heterogeneous materials. A comprehensive overview of different homogenization methods can be found in Reference [1].

Among those different methods, the mean-field homogenization (MFH) approaches provide predictions for the macroscopic behavior of heterogeneous materials at a reasonable computational cost. They were first developed for linear elastic problems as semi-analytical methods based on the Eshelby single inclusion solution [2]. To account for the interactions between inclusions in an average way, additional assumptions are applied as in the Mori-Tanaka scheme $[3,4]$ or in the self-consistent scheme [5-8].

When extending MFH methods to the non-linear regime, most approaches revolve around the definition of a so-called linear comparison composite (LCC) $[9,10]$, which is a virtual composite whose constituents linear behaviors match the linearized behavior of the real composite constituents for a given strain state. The use of a LCC allows the available linear schemes being applied to non-linear composites [11-13]. Such a LCC is used in the incremental formulation proposed by Hill [14], which considers linearized relations between the stress and strain increments of the different constituents around their current strain states, and for which the constitutive equations of the homogenized structure are rewritten in a pseudo-linear form relating the stress and strain rates. This incremental formulation was further developed by several authors in order to improve its predictive capabilities [15-18]. In particular, by considering the isotropic part of the tangent operator in computing Eshelby or Hill tensors, very acceptable estimates of the macroscopic response were obtained for different non-linear constitutive relations.

For completeness, other homogenization techniques can be used in the nonlinear range as the computational homogenization method that was developed to account for complex non-linear behaviors at the micro-scale [19, 20].

At the current state-of-the-art, multiscale homogenization methods in general and mean-field homogenization schemes in particular can predict accurately the macroscopic behavior of heterogeneous materials exhibiting non-linear irreversible behaviors at the microscopic components scale. However, capturing the degradation, damage or failure of material happening at the microscopic scale remains challenging when dealing with multiscale methodologies. Besides the complexity of formulating such a multiscale method, when continuum damage models are applied to capture degradation and failure of materials, at the micro or macro scale, the governing partial differential equations may lose ellipticity at a given level of loading corresponding to the strain-softening onset. Consequently, the boundary or initial value problem becomes ill-posed, and the associated finite element simulations suffer from the loss of uniqueness and from the strain localization problem. Once loss of uniqueness happens, the numerical 
solutions become meaningless and differ with the size and direction of the mesh without convergence.

Many enhanced physical or phenomenological models are proposed to overcome this difficulty. Commonly the continuum modeling is supplied with a higher-order formulation, such as in the Cosserat model [21], the non-local model [22] or the gradient model [23]. Interactions between neighboring material points are reflected in these models through the internal length, which is related to the microstructure and the failure mechanisms of the material. An overview of these methods can be found in Reference [24]. In order to avoid the necessity of developing elements with higher-order derivatives, required to evaluate explicitly internal variable derivatives, such as strain gradient, the nonlocal kernel has been reformulated in an implicit way such that a new non-local variable, representative of an internal variable and its derivatives, results from the resolution of a new boundary value problem [25-27].

Although the higher-order and non-local formulations mentioned above have been widely used to avoid losing ellipticity at strain-softening onset, their applications in multi-scale computations are rare. Liu and $\mathrm{Hu}$ [28] applied the Cosserat model in Mori-Tanaka procedure to study the particle size dependence of composite materials. Dascalu [29] connected the locally periodic micro-crack with the macroscopic damage through asymptotic developments homogenization. Knockaert and Doghri [30] introduced the gradients of the internal variables, which are obtained from a micro/macro homogenization procedure, at the macro-scale computation. However, the resulting formulation was only accomplished in 1D case because of the difficulties in implementation. Massart et al. [31, 32] introduced the non-local approach in the framework of the computational homogenization for the problem of masonry. In this work, the finite element resolution at the micro(meso)-scale is based on a non-local implicit approach, while micro-macro continuous/discontinuous up-scaling allows treating the localization at the macro-scale by means of embedded bands. More recently, Coehnen et al. [33, 34] extended this method in a more general setting. However, a multiscale analysis allowing to capture softening at the micro-scale and at the macro-scale without causing localization based on (semi-)analytical homogenization methods is still missing. Although less accurate than when relying on a computational homogenization, such an approach would benefit from reduced computational costs.

This paper investigates the application of ductile-damage theories to a multiscale analysis of continuous fiber reinforced composites. Toward this end, the incremental MFH approach is extended to account for the damage behavior happening in the matrix material at the micro-scale and to derive the effective properties of particle or fiber reinforced composites. In order to avoid the strain/damage localization caused by the matrix material softening, the gradient-enhanced formulation [35] is adopted to describe the material behavior of the matrix. As a result this new formulation allows simulating the plylevel response under quasi-static loading conditions resulting from the coupled plasticity-damage model considered at the matrix micro-scale.

The paper is organized as follows. As a prerequisite to the development of the 
multiscale analysis with a gradient-enhanced damage model, section 2 summarizes the state-of-the-art of mean field homogenization methods for two-phase elasto-plastic composite and the derivation of the gradient-enhanced damage model for continuum mechanics. Section 3 presents the new multiscale analysis, with in particular the new mean-field homogenization scheme with the gradient-enhanced damage model at the micro-scale, the induced modification of the macroscopic formulation which involves a new boundary value problem to be solved, and its finite-element implementation. Numerical studies are conducted in section 4 . In particular studies comparing the developed method with respect to the direct numerical simulation of a unit cell and of a representative volume element (RVE) are achieved, demonstrating the ability of the method to capture the composite behavior for low to moderate fiber volume ratios. Also a multiscale simulation at the ply level is conducted. In this simulation, the ply is notched inducing a damage localization, which is shown to be independent of the macroscopic mesh considered. Finally, conclusions are gathered in section 5.

\section{Scientific background}

In this section, the prerequisites to the development of a new multiscale analysis with a gradient-enhanced damage model are summarized. In particular, the main equations of the mean field homogenization method for two-phase elasto-plastic composites are derived according to the incremental formulations considering the material laws in a pseudo-linear form relating the stress and strain rates. Also, with a view to the development of the non-local multiscale method, the main features of the gradient-enhanced damage model are recalled and applied to the Lemaitre-Chaboche ductile damage model.

\subsection{Mean field homogenization for two-phase elasto-plastic composites}

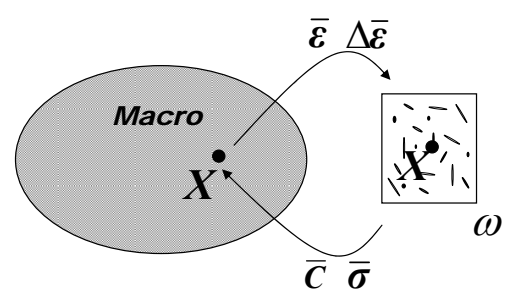

Figure 1: Multiscale method.

In a multiscale approach, at each macro-point $\boldsymbol{X}$, the macro-strain $\bar{\varepsilon}$ is known, and the macro-stress $\overline{\boldsymbol{\sigma}}$ is sought from a micro-scale boundary value problem (BVP), or vice-versa. At the micro-level, the macro-point is viewed as the center of a RVE of domain $x \in \omega$ and boundary $\partial \omega$. This multiscale procedure is illustrated in Fig. 1. Considering adequate boundary conditions 
(BCs) on the RVE, the Hill-Mandell condition, expressing the equality between energies at both scales, transforms the relation between macro-strains $\bar{\varepsilon}$ and stresses $\overline{\boldsymbol{\sigma}}$ into the relation between average strains $\langle\boldsymbol{\varepsilon}\rangle$ and stresses $\langle\boldsymbol{\sigma}\rangle$ over the RVE, with

$$
\bar{\varepsilon}=\langle\varepsilon\rangle \quad \text { and } \quad \overline{\boldsymbol{\sigma}}=\langle\boldsymbol{\sigma}\rangle,
$$

where $\langle f(\boldsymbol{x})\rangle=\frac{1}{V_{\omega}} \int_{\omega} f(\boldsymbol{x}) \mathrm{d} V$.

Considering a two-phase isothermal linear elastic composite, with uniform constitutive material stiffness tensors $\boldsymbol{C}_{0}$ for the matrix and $\boldsymbol{C}_{\mathrm{I}}$ for the inclusions, and with the respective volume fractions $v_{0}+v_{\mathrm{I}}=1$ (subscript 0 refers to the matrix and I to the inclusions), Eq. (1) can be rewritten in the matrix subdomain $\omega_{0}$ and in the inclusions subdomain $\omega_{\mathrm{I}}$ as

$$
\overline{\boldsymbol{\varepsilon}}=v_{0}\langle\boldsymbol{\varepsilon}\rangle_{\omega_{0}}+v_{\mathrm{I}}\langle\boldsymbol{\varepsilon}\rangle_{\omega_{\mathrm{I}}} \quad \text { and } \quad \overline{\boldsymbol{\sigma}}=v_{0}\langle\boldsymbol{\sigma}\rangle_{\omega_{0}}+v_{\mathrm{I}}\langle\boldsymbol{\sigma}\rangle_{\omega_{\mathrm{I}}}
$$

The system of Eqs. (2) is completed by an equation describing the relation between the strain averages per phase through a strain concentration tensor $\boldsymbol{B}^{\epsilon}$ :

$$
\langle\varepsilon\rangle_{\omega_{\mathrm{I}}}=\boldsymbol{B}^{\epsilon}:\langle\varepsilon\rangle_{\omega_{0}}
$$

To express this strain concentration tensor $\boldsymbol{B}^{\epsilon}$, assumptions should be made on the micro-mechanics, and different $\mathrm{MFH}$ models give different expressions of $\boldsymbol{B}^{\epsilon},[3,8]$, all of them being an approximate solution. In this paper, we consider the Mori-Tanaka (M-T) model because it provides good predictions for twophase composite materials for which the matrix can be clearly identified [36], and has been shown to be accurate for low to moderate fiber volume fractions. This model assumes that the strain at infinity for the single inclusion problem corresponds to the average strain in the matrix phase of the multiple inclusions composite. In this case, the strain concentration tensor reads

$$
\boldsymbol{B}^{\epsilon}=\left\{\boldsymbol{I}+\boldsymbol{S}:\left[\left(\boldsymbol{C}_{0}\right)^{-1}: \boldsymbol{C}_{\mathrm{I}}-\boldsymbol{I}\right]\right\}^{-1},
$$

where the Eshelby tensor $\boldsymbol{S}\left(\mathrm{I}, \boldsymbol{C}_{0}\right)$ depends on the geometry of the inclusion (I) and on $\boldsymbol{C}_{0}[2]$. Thus the macroscopic material behavior can be written in the form

$$
\overline{\boldsymbol{\sigma}}=\overline{\boldsymbol{C}}: \overline{\boldsymbol{\varepsilon}}, \text { with } \overline{\boldsymbol{C}}=\left[v_{\mathrm{I}} \boldsymbol{C}_{\mathrm{I}}: \boldsymbol{B}^{\epsilon}+v_{0} \boldsymbol{C}_{0}\right]:\left[v_{\mathrm{I}} \boldsymbol{B}^{\epsilon}+v_{0} \boldsymbol{I}\right]^{-1},
$$

where $\bar{C}$ is the homogenized macro-stiffness tensor ${ }^{1}$.

For two-phase elasto-plastic composites, the MFH methods revolve around the introduction of a so-called linear comparison composite (LCC), which allows the linear schemes being applied to non-linear behaviors. Different linearizations lead to various formulations, such as the secant (or total) deformation formulation [37, 38], the incremental formulation [14], the variational formulation

\footnotetext{
${ }^{1}$ Mathematical notations are defined in Appendix A.
} 
[39-42] or the affine formulation [43, 44]. Because of its simplicity the incremental formulation is considered in this paper motivating a brief review of its basic theory.

During a finite incremental process, the constitutive equations, of the two phases, are discretized in time intervals $\left[t_{n}, t_{n+1}\right]$, and the expression equivalent to (5) now relates the macroscopic homogenized stress increment $\Delta \overline{\boldsymbol{\sigma}}$ to the macroscopic homogenized strain increment $\Delta \bar{\varepsilon}$ through

$$
\Delta \bar{\sigma}=\bar{C}: \Delta \bar{\varepsilon}
$$

where $\overline{\boldsymbol{C}}$ is the tensor of macro-moduli of the homogenized elasto-plastic twophase composite to be found. Toward this end, the macro linearizations of strain and stress tensors (2) are obtained from the average linearizations of strain and stress in the phases. When considering a finite increment $\Delta$ on the time interval $\left[t_{n}, t_{n+1}\right]$, these relations read

$$
\begin{aligned}
\Delta \bar{\varepsilon} & =v_{0}\langle\Delta \varepsilon\rangle_{\omega_{0}}+v_{\mathrm{I}}\langle\Delta \boldsymbol{\varepsilon}\rangle_{\omega_{\mathrm{I}}}, \quad \text { and } \\
\Delta \overline{\boldsymbol{\sigma}} & =v_{0}\langle\Delta \boldsymbol{\sigma}\rangle_{\omega_{0}}+v_{\mathrm{I}}\langle\Delta \boldsymbol{\sigma}\rangle_{\omega_{\mathrm{I}}} .
\end{aligned}
$$

At this point, considering the so-called linear comparison composite, the relation between the average incremental strains in the two phases (3) is rewritten from the "consistent" tangent operators $\boldsymbol{C}_{0}^{\text {alg }}$ of the matrix phase and $\boldsymbol{C}_{\mathrm{I}}^{\text {alg }}$ of the inclusions phase, leading to

$$
\langle\Delta \varepsilon\rangle_{\omega_{\mathrm{I}}}=\boldsymbol{B}^{\epsilon}\left(\boldsymbol{I}, \overline{\boldsymbol{C}}_{0}^{\mathrm{alg}}, \overline{\boldsymbol{C}}_{\mathrm{I}}^{\mathrm{alg}}\right):\langle\Delta \varepsilon\rangle_{\omega_{0}}
$$

The tensor $\overline{\boldsymbol{C}}_{0}^{\text {alg }}$ (respectively $\overline{\boldsymbol{C}}_{\mathrm{I}}^{\text {alg }}$ ) is computed from the material model of the matrix (respectively inclusions) phase using $\langle\Delta \varepsilon\rangle_{\omega_{0}}$ (respectively $\langle\Delta \varepsilon\rangle_{\omega_{\mathrm{I}}}$ ). Thus, $\overline{\boldsymbol{C}}_{0}^{\text {alg }}$ and $\overline{\boldsymbol{C}}_{\mathrm{I}}^{\text {alg }}$ are uniform by construction, and called comparison tangent operators; Finally, similarly to the elastic counter part (5), the macroscopic homogenized stress increment $\Delta \overline{\boldsymbol{\sigma}}$ can be related to the macroscopic homogenized strain increment $\Delta \bar{\varepsilon}$ through (6) with the macro-moduli tensor of the homogenized elasto-plastic two-phase composite evaluated from

$$
\overline{\boldsymbol{C}}=\left[v_{\mathrm{I}} \overline{\boldsymbol{C}}_{\mathrm{I}}^{\mathrm{alg}}: \boldsymbol{B}^{\epsilon}+v_{0} \overline{\boldsymbol{C}}_{0}^{\mathrm{alg}}\right]:\left[v_{\mathrm{I}} \boldsymbol{B}^{\epsilon}+v_{0} \boldsymbol{I}\right]^{-1} .
$$

Note, that when computing the Eshelby tensor $\boldsymbol{S}\left(I, \overline{\boldsymbol{C}}_{0}^{\text {iso }}\right)$ required to compute the Mori-Tanaka strain concentration tensor (4), only the isotropic part $\overline{\boldsymbol{C}}_{0}^{\text {iso }}$ of $\overline{\boldsymbol{C}}_{0}^{\text {alg }}$ is applied to improve prediction results. More details can be found in reference [44].

For completeness, when solving the problem at the finite element level using a Newton-Raphson procedure, one needs to linearize the stress tensor at time $t_{n+1}$ yielding

$$
\delta \overline{\boldsymbol{\sigma}}_{n+1}=\overline{\boldsymbol{C}}^{\mathrm{alg}}: \delta \overline{\boldsymbol{\varepsilon}}_{n+1},
$$

where $\overline{\boldsymbol{C}}^{\text {alg }}$ is the Jacobian operator obtained during the MFH process as described in section 3.1.1. 
This method has been shown to be very accurate for 2-phase composites with moderate fiber volume ratios, and for which, at the RVE size, the matrix can be considered as homogeneous isotropic and the ellipsoidal fibers can be considered as homogeneous transversely isotropic.

\subsection{The non-local gradient model}

In order to avoid the loss of solution uniqueness and the strain localization problem when extending MFH to damage theories, the model should be written in a non-local form involving gradient enrichments. Toward this end, some internal variables $a$ (which can be strains, accumulated plastic strain, damage...) are replaced by their weighted average $\tilde{a}$ over a characteristic volume $\left(V_{C}\right)$ to reflect the interaction between neighboring material points [45]:

$$
\tilde{a}(\boldsymbol{X})=\frac{1}{V_{C}} \int_{V_{C}} a(\boldsymbol{y}) \phi(\boldsymbol{y} ; \boldsymbol{X}) \mathrm{d} V,
$$

where $\boldsymbol{y}$ denotes positions inside the characteristic volume $V_{C}$. The weight function $\phi(\boldsymbol{y} ; \boldsymbol{X})$ determines the influence of the local internal variables in the characteristic volume $V_{C}$ on the non-local internal variable at point $\boldsymbol{X}$. For convenience, the weight functions are assumed normalized such that one has $\frac{1}{V_{C}} \int_{V_{C}} \phi(\boldsymbol{y} ; \boldsymbol{X}) \mathrm{d} V=1$.

Practically, in order to avoid the direct computation of (12) in a finite element framework, this expression can be substituted by an explicit approximated solution, or by an implicit form preserving the high order accuracy.

\subsubsection{Explicit gradient formulation}

For a sufficiently smooth field $a$ and when taken sufficiently far from the boundaries, the integral relation (12) can be rewritten in terms of the evenorder gradients of $a$ by expanding the field into Taylor series [27]. Odd orders annihilate due to the symmetry of the weight functions. Neglecting terms of fourth-order and higher, the integral relation (12) is approximated by the differential relation

$$
\tilde{a}(\boldsymbol{X})=a(\boldsymbol{X})+l^{2} \nabla^{2} a(\boldsymbol{X}),
$$

where the Laplacian operator $\nabla^{2} a(\boldsymbol{X})=\sum_{i} \partial^{2} / \partial X_{i}^{2} a$. The internal length scale of the non-local model is preserved in the gradient coefficient $l$. On top of the difficulty of applying adequate boundary conditions, the numerical evaluation of relation (13) in a traditional finite element framework is not straightforward as elements are usually not satisfying high order continuities. These drawbacks motivated the development of the so-called implicit method [27].

\subsubsection{Implicit gradient formulation}

Considering Green functions $G(\boldsymbol{y} ; \boldsymbol{X})$ as weighting functions $\phi(\boldsymbol{y} ; \boldsymbol{X})$ in (12), an alternative second-order gradient approximation ${ }^{2}$ can be derived after some

\footnotetext{
${ }^{2}$ Here the approximation results from the particular choice of the weighting functions only
} 
further mathematical manipulations [27], leading to

$$
\tilde{a}(\boldsymbol{X})-l^{2} \nabla^{2} \tilde{a}(\boldsymbol{X})=a(\boldsymbol{X})
$$

where the Laplacian operator is defined as $\nabla^{2} \tilde{a}(\boldsymbol{X})=\sum_{i} \partial^{2} / \partial X_{i}^{2} \tilde{a}$. In contrast to the definition (13), the non-local internal variable $\tilde{a}$ is not given explicitly in terms of $a$ and its derivatives, but is the solution of a new boundary value problem consisting of the Helmholtz equation (14) completed by appropriate boundary conditions.

By opposition to the explicit form (13), this gradient model (14) is truly non-local in the sense that variations of the local internal variable $a$ in the neighboring of $\boldsymbol{X}$ always affect the non-local internal variable $\tilde{a}(\boldsymbol{X})$, due to the definition of the Green functions and as equation (14) is satisfied exactly during the computation. Another advantage of (14) compared to the explicit formulation is that it can be implemented without recourse to high-order finiteelements and that it avoids the consideration of complex boundary conditions on the non-local variable, see [27] for a complete discussion.

\subsubsection{Boundary conditions}

As non-local formulations involve higher order derivatives, additional boundary conditions are required to have a well-posed problem. For the implicit gradient model based on equation (14), the natural boundary conditions usually considered are [35]

$$
\frac{\partial \tilde{a}}{\partial n}=n_{i} \frac{\partial \tilde{a}}{\partial X_{i}}=0,
$$

where $\boldsymbol{n}$ is the outward unit normal. Indeed, these boundary conditions lead to a behavior that is consistent with relation (12), i.e.

- $\tilde{a}$ always equals $a$ for homogeneous deformations;

- The total internal variable content is preserved in the non-local averaging: $\int_{\Omega} \tilde{a} \mathrm{~d} \Omega=\int_{\Omega} a \mathrm{~d} \Omega$, where $\Omega$ is the problem domain.

Note that the finite-element formulation of the implicit formulation naturally includes these boundary conditions, which, thus, do not need to be defined by the user.

\subsubsection{Application to the Lemaitre - Chaboche ductile damage model}

In particular, the non-local implicit approach can be applied to damage models in order to avoid any loss of ellipticity. In this paper, we only consider the damage in the isotropic matrix. In order to simplify the developments of the method, an isotropic damage model is assumed. Although to remain more general, a damage variable should be represented by a fourth order tensor due to the existence of several mechanisms of damage, this is not necessary for damage induced by meso- or micro-plasticity [46], which justifies the use of a simple model for the matrix material. In this work, we focus on the Lemaitre - 
Chaboche ductile damage model [46]. The damage is introduced with the usual assumption that the strain observed in the actual body and in its undamaged representation are equivalent $[47,48]$, and the usual definition of the average effective stress reads

$$
\hat{\boldsymbol{\sigma}}=\frac{\boldsymbol{\sigma}}{(1-D)},
$$

where $0 \leq D<1$ is the damage variable.

Assuming an elasto-plastic material, which obeys $J_{2}$ elasto-plasticity, the von Mises stress criterion reads

$$
f=\hat{\sigma}^{e q}-R(p)-\sigma_{\mathrm{Y}} \leqslant 0 .
$$

In this expression, $f$ is the yield surface, $\hat{\sigma}^{\mathrm{eq}}=\sqrt{\frac{3}{2} \frac{\operatorname{dev}(\boldsymbol{\sigma})}{1-D}: \frac{\operatorname{dev}(\boldsymbol{\sigma})}{1-D}}$ is the equivalent von Mises effective stress, $\sigma_{\mathrm{Y}}$ is the initial yield stress, $R(p) \geqslant 0$ is the isotropic hardening stress, and $p$ is an internal variable characterizing the irreversible behavior. If $f<0$, the behavior remains elastic. On the other hand, if $f=0$, then $\dot{p}$ is positive and the plastic strain tensor increment obeys the normal plastic flow, which is summarized by

$$
\dot{\boldsymbol{\varepsilon}}^{\mathrm{pl}}=\dot{p} \boldsymbol{N}, \quad \text { with } \boldsymbol{N}=\frac{\partial f}{\partial \hat{\boldsymbol{\sigma}}}=\frac{3}{2} \frac{\operatorname{dev}(\boldsymbol{\sigma})}{(1-D) \hat{\sigma}^{\mathrm{eq}}},
$$

where $N$ is the normal to the yield surface in the effective stress space. In this formalism, the internal variable $p$ represents the accumulated plastic strain $\dot{p}=\left[\frac{2}{3} \dot{\varepsilon}^{\mathrm{pl}}: \dot{\boldsymbol{\varepsilon}}^{\mathrm{pl}}\right]^{1 / 2}$, and, assuming small deformations, the reversible (elastic) and irreversible (plastic) strain tensors can be added $\left(\varepsilon=\varepsilon^{\mathrm{el}}+\varepsilon^{\mathrm{pl}}\right)$, yielding

$$
\boldsymbol{\sigma}=(1-D) \boldsymbol{C}^{\mathrm{el}}:\left(\varepsilon-\varepsilon^{\mathrm{pl}}\right),
$$

where $\boldsymbol{C}^{\mathrm{el}}$ is the fourth-order Hooke tensor of the sane (undamaged) material.

What remains to be defined is the evolution of the damage with $\dot{p}$. In this paper, we focus on the Lemaitre-Chaboche ductile damage model [46]. Following the technique proposed in [49] to develop non-local damage laws, in this paper the non-local accumulated plastic strain $\tilde{p}$ is applied to calculate the damage evolution in the Lemaitre-Chaboche model:

$$
\dot{D}= \begin{cases}0, & \text { if } \tilde{p} \leqslant p_{C} \\ \left(\frac{Y}{S_{0}}\right)^{s} \dot{\tilde{p}}, & \text { if } \tilde{p}>p_{C} .\end{cases}
$$

In this expression, $p_{C}$ is a plastic threshold for the evolution of damage, $S_{0}$ and $s$ are the material parameters, and $Y$ is the strain energy release rate computed as

$$
Y=\frac{1}{2} \varepsilon^{\mathrm{el}}: C^{\mathrm{el}}: \varepsilon^{\mathrm{el}} .
$$

Eventually the damage can simply be obtained from the time-integration of (20): $D=\int \dot{D} \mathrm{~d} t$. 
In this gradient enhanced damage model, the non-local accumulated plastic strain $\tilde{p}$ is computed from the implicit formulation (14), which becomes

$$
\tilde{p}-l^{2} \nabla^{2} \tilde{p}=p .
$$

In this formalism, the length scale $l$ is related to the characteristic microstructure length. The local accumulated plastic strain in Eq. (22) is still computed from the plastic flow (18). The equations governing the $J_{2}$-plasticity, i.e. Eqs. (17-18) involve explicitly the local accumulated plastic strain $p$ only, while the non-local form $\tilde{p}$ appears in (20) only. Indeed, as demonstrated in [35, 49], considering the non-local form $\tilde{p}$ in the definition of the yield surface would lead to unphysical results, such as a constant plastic strain on the whole domain.

During a finite incremental process, the constitutive equations are discretized in time intervals $\left[t_{n}, t_{n+1}\right]$, and the constitutive equations are differentiated at $t_{n+1}$. Remembering that for the non-local formulation the damage depends on both $\varepsilon$ and on $\tilde{p}$, this linearization reads

$$
\delta \boldsymbol{\sigma}_{n+1}=\boldsymbol{C}^{\mathrm{algD}}: \delta \boldsymbol{\varepsilon}_{n+1}-\left(\hat{\boldsymbol{\sigma}} \otimes \frac{\partial D}{\partial \boldsymbol{\varepsilon}}\right): \delta \boldsymbol{\varepsilon}_{n+1}-\hat{\boldsymbol{\sigma}} \frac{\partial D}{\partial \tilde{p}} \delta \tilde{p}_{n+1},
$$

where

$$
\boldsymbol{C}^{\mathrm{algD}}=(1-D) \boldsymbol{C}^{\mathrm{alg}} .
$$

In these last expressions, $C^{\text {alg }}$ is the derivative of the effective stress increment with respect to the strain increment ${ }^{3}$, [50]. Its expression, and the explicit expressions of the damage derivatives are given in Appendix B.

\section{Multiscale analysis a with gradient-enhanced damage model}

The purpose of this section is to develop a multiscale model for 2-phase composites where the matrix material obeys an elasto-plastic behavior experiencing damage. Toward this end, the matrix material behavior is described by the gradient-enhanced damage model presented in section 2.2.4. The multiscale analysis is based on an incremental MFH scheme, as presented in section 2.1, which is extended herein to account for the non-local damage model of the matrix. The fibers are assumed to remain elastic. As considering the implicit formulation of the gradient-enhanced damage model implies solving new Helmholtz-type equations, the macro-scale governing equations have to be completed to fully determine the micro-macro transition. Finally the finite-element implementation of this MFH-based multiscale framework is derived.

\footnotetext{
${ }^{3}$ In this non-local formalism, we explicitly write the dependence of the stress in $D$ and $\varepsilon$ and linearize with respect to both terms. We use symbol $\boldsymbol{C}^{\text {alg }}$ for the derivative of the effective stress increment with respect to the strain increment.
} 


\subsection{The gradient-enhanced damage model in the micro-scale analysis}

In this section we consider a finite incremental process for which the constitutive equations are discretized in time intervals $\left[t_{n}, t_{n+1}\right]$. Unless specified otherwise, variables are referred to their value at time $t_{n+1}$ and their linearization at that time is denoted using symbol $\delta$. The average stress and strain tensors in the RVE are respectively denoted $\overline{\boldsymbol{\sigma}}$ and $\overline{\boldsymbol{\varepsilon}}$. The average micro-stress and micros-strain tensors in each phase, previously referred to as, respectively, $\langle\boldsymbol{\sigma}\rangle_{\omega_{\mathrm{i}}}$ and $\langle\varepsilon\rangle_{\omega_{\mathrm{i}}}$, are now, respectively, denoted $\boldsymbol{\sigma}_{\mathrm{i}}$ and $\varepsilon_{\mathrm{i}}$ to simplify the equations.

\subsubsection{MFH with a gradient-enhanced damage model}

Following the incremental formulation of MFH presented in section 2.1, the stress linearization reads:

$$
\delta \overline{\boldsymbol{\sigma}}=v_{0} \delta \boldsymbol{\sigma}_{0}+v_{\mathrm{I}} \delta \sigma_{\mathrm{I}}
$$

The behavior of the fibers is assumed to remain elastic, and $\delta \boldsymbol{\sigma}_{\mathrm{I}}=\overline{\boldsymbol{C}}_{\mathrm{I}}^{\mathrm{el}}: \delta \boldsymbol{\varepsilon}_{\mathrm{I}}$. The matrix obeys the non-local damage elasto-plastic behavior described in section 2.2.4, and equation (23) allows writing

$$
\delta \boldsymbol{\sigma}_{0}=\overline{\boldsymbol{C}}_{0}^{\mathrm{alg} D}: \delta \boldsymbol{\varepsilon}_{0}-\left(\hat{\boldsymbol{\sigma}}_{0} \otimes \frac{\partial D}{\partial \boldsymbol{\varepsilon}_{0}}\right): \delta \boldsymbol{\varepsilon}_{0}-\hat{\boldsymbol{\sigma}}_{0} \frac{\partial D}{\partial \tilde{p}} \delta \tilde{p} .
$$

However, as this last equation involves average stress and strain tensors on the matrix phase, one should be careful on the meaning of $D$ and $\tilde{p}$ which are representations of, respectively, the damage and the non-local equivalent plastic strain in the matrix of the RVE and do not correspond to the average values ${ }^{4}$. Thus, eq. (25) becomes

$$
\delta \overline{\boldsymbol{\sigma}}=v_{\mathrm{I}} \overline{\boldsymbol{C}}_{\mathrm{I}}^{\mathrm{alg}}: \delta \varepsilon_{\mathrm{I}}+v_{0} \overline{\boldsymbol{C}}_{0}^{\mathrm{algD}}: \delta \boldsymbol{\varepsilon}_{0}-v_{0}\left(\hat{\boldsymbol{\sigma}}_{0} \otimes \frac{\partial D}{\partial \boldsymbol{\varepsilon}_{0}}\right): \delta \varepsilon_{0}-v_{0} \hat{\boldsymbol{\sigma}}_{0} \frac{\partial D}{\partial \tilde{p}} \delta \tilde{p}
$$

where the operators of the matrix $\overline{\boldsymbol{C}}_{0}^{\text {algD }}$, and of the fibers $\overline{\boldsymbol{C}}_{\mathrm{I}}^{\text {alg }}=\overline{\boldsymbol{C}}_{\mathrm{I}}^{\mathrm{el}}$, which are by construction uniform, are designated as "comparison" operators in the MFH literature.

The first two terms at the right hand side of Eq. (27) represent the stress variation of a composite with a fictitious matrix material of uniform non-local damage. As this non-local damage is a non-local representation of an internal variable of the matrix only, we assume that these two terms define a Linear Comparison Composite (LCC) on which the incremental Mori-Tanaka process can be applied. Indeed, the last two terms of Eq. (27) are related to the

\footnotetext{
${ }^{4}$ Indeed, starting from $\boldsymbol{\sigma}=(1-D) \hat{\boldsymbol{\sigma}}$, the average tensor $\langle\delta \boldsymbol{\sigma}\rangle_{\omega_{0}}=\langle(1-D) \delta \hat{\boldsymbol{\sigma}}\rangle_{\omega_{0}}-\langle\hat{\boldsymbol{\sigma}} \delta D\rangle_{\omega_{0}}$ can be written as $\langle\delta \boldsymbol{\sigma}\rangle_{\omega_{0}}=\left(1-D^{*}\right)\langle\delta \hat{\boldsymbol{\sigma}}\rangle_{\omega_{0}}-\langle\hat{\boldsymbol{\sigma}}\rangle_{\omega_{0}} \delta D^{*}$, where $D^{*} \neq\langle D\rangle_{\omega_{0}}$ is a representation of the damage of the matrix. For simplicity, we do not use $D^{*}$ but $D$ in the developments of this section.
} 
softening of the matrix due to the damage. By choice, they are not considered when defining the LCC for the M-T process, and the slope $(1-D) \overline{\boldsymbol{C}}_{0}^{\text {alg }}$ for the damaged matrix phase is used for two reasons. On the one hand, M-T is only defined when the two tangent moduli of the LCC are definite positive, which would not be the case in the softening part if these two terms were considered. Note that if considering another approach than the incremental formulation, such as the affine formulation or the transformation field analysis $[51,52]$, this limitation could be removed. In the transformation field analysis $[51,52]$ for elasto-plastic composites, a relaxation stress is defined due to the irreversible behavior in the matrix. In our approach the relaxation stress, due to plasticity and damage, is partly modeled by using the damaged elasto-plastic modulus of matrix $\overline{\boldsymbol{C}}_{0}^{\mathrm{algD}}$ in the M-T process, see details here below. However, the relaxation stress resulting from the softening in the matrix (last two terms of Eq. (27)) is omitted due to our approximation. On the other hand, neglecting the last two terms of Eq. (27) introduces an error as the softening of the matrix does not lead to an unloading of fibers. However, this is an error of the same order of magnitude as for elasto-plastic behaviors. Indeed MFH using the LCC incremental formulation does not unload the stress in the fibers reached at the end of the previous stress increment. This correction should result from a modification of the material stiffness. Thus we expect our M-T based MFH to predict stresses slightly above the solution obtained with a direct finite element simulation.

Considering a time step $\left[t_{n}, t_{n+1}\right]$, the known data are the macro-total strain tensor $\bar{\varepsilon}_{n}$, the strain increment $\Delta \bar{\varepsilon}_{n+1}$ and the non-local accumulated plastic strain increment $\Delta \tilde{p}_{n+1}$ of the matrix material, as well as the internal variables at $t_{n}$. The set of non-linear equations to be solved can thus be stated as

$$
\begin{aligned}
\Delta \overline{\boldsymbol{\varepsilon}}_{n+1} & =v_{0} \Delta \varepsilon_{0 n+1}+v_{\mathrm{I}} \Delta \varepsilon_{\mathrm{I} n+1} \\
\overline{\boldsymbol{\sigma}}_{n+1} & =v_{0} \boldsymbol{\sigma}_{0 n+1}+v_{\mathrm{I}} \boldsymbol{\sigma}_{\mathrm{I} n+1} \\
\Delta \varepsilon_{\mathrm{I} n+1} & =\boldsymbol{B}^{\epsilon}\left(\boldsymbol{I}, \overline{\boldsymbol{C}}_{0}^{\mathrm{algD}}, \overline{\boldsymbol{C}}_{\mathrm{I}}^{\text {alg }}\right): \Delta \varepsilon_{0 n+1}
\end{aligned}
$$

where $\boldsymbol{\sigma}_{0 n+1}$ (respectively $\boldsymbol{\sigma}_{\mathrm{In}+1}$ ) is evaluated from $\Delta \boldsymbol{\varepsilon}_{0 n+1}$ and $\Delta \tilde{p}_{n+1}$ (respectively $\left.\Delta \varepsilon_{\mathrm{I}_{n+1}}\right)$ using the non-local Lemaitre - Chaboche ductile damage model described in section 2.2.4 (respectively the elastic constitutive behavior).

Practically, this system of equations is solved using the following M-T process:

- Predict Eshelby tensor $\boldsymbol{S}\left(\boldsymbol{I}, \overline{\boldsymbol{C}}_{0_{n}}^{\text {isoD }}\right)$ using the isotropic moduli of $\overline{\boldsymbol{C}}_{0}^{\text {algD }}$ of matrix at $t_{n}{ }^{5}$.

- Initialize the strain increment in inclusions: $\Delta \bar{\varepsilon}_{n+1} \rightarrow \Delta \varepsilon_{\text {In+1 }}$.

\footnotetext{
${ }^{5}$ We use $\overline{\boldsymbol{C}}_{0}^{\text {isoD }}$ evaluated at previous time step to compute the Eshelby tensor to avoid the need of accounting for the derivation of $\boldsymbol{S}$ during the iteration process that follows.
} 
- Follow the iterations process (upper indices $(i)$ for values at iteration $i$ of time $t_{n+1}$ are omitted for simplicity):

1. Call the constitutive material function, here considered elastic, of the real inclusions material with, as inputs, the average strain tensor and the increment in the inclusions phase, $\varepsilon_{\mathrm{In} n}$ and $\Delta \varepsilon_{\mathrm{I} n+1}$. The material function returns the updated stress $\sigma_{\mathrm{I}_{n+1}}$, and the algorithmic moduli $\overline{\boldsymbol{C}}_{\mathrm{I}}^{\mathrm{alg}}{ }_{n+1}$, here Hooke tensor, for the inclusion phase.

2. Compute the average strain in the matrix phase:

$$
\Delta \varepsilon_{0 n+1}=\left(\Delta \bar{\varepsilon}_{n+1}-v_{\mathrm{I}} \Delta \varepsilon_{\mathrm{In}+1}\right) / v_{0} .
$$

3. Call the constitutive material function of the real matrix material with, as inputs, the average strain tensor and increment in the matrix phase, $\varepsilon_{0 n}, \Delta \varepsilon_{0 n+1}$, the non-local accumulated plastic strain increment $\Delta \tilde{p}_{n+1}$, and the internal variables at time $t_{n}$. First evaluate at time $t_{n}$ the effective average stress in the matrix, as

$$
\hat{\boldsymbol{\sigma}}_{0 n}=\boldsymbol{\sigma}_{0 n} /\left(1-D_{n}\right),
$$

and then evaluate the effective trial stress tensor, which will be used in the radial return mapping process, as

$$
\hat{\boldsymbol{\sigma}}_{0 n+1}^{\mathrm{tr}}=\hat{\boldsymbol{\sigma}}_{0 n}+\boldsymbol{C}_{0}^{\mathrm{el}}: \Delta \boldsymbol{\varepsilon}_{0 n+1} .
$$

After having applied the radial return mapping, the output is the updated stress $\boldsymbol{\sigma}_{0 n+1}$, the internal variables at time $t_{n+1}$, including the damage variable $D_{n+1}$ and the (anisotropic) moduli $\overline{\boldsymbol{C}}_{0}^{\text {alg }}{ }_{n+1}$ for the matrix phase without damage.

4. In case of damage evolution compute $\overline{\boldsymbol{C}}_{0}^{\text {algD }}{ }_{n+1}=\left(1-D_{n+1}\right) \overline{\boldsymbol{C}}_{0}^{\text {alg }}{ }_{n+1}$.

5. Extract $\overline{\boldsymbol{C}}_{0}^{\text {isoD }}{ }_{n+1}$, the isotropic part of $\overline{\boldsymbol{C}}_{0}^{\text {algD }}{ }_{n+1}$ for further evaluation of the Eshelby tensor at next time step.

6. As for improving robustness and accuracy, the MFH process can be conducted at time $t_{n+\alpha}$, with $\alpha=1 / 2$ (mid-point rule) or $\alpha=2 / 3^{6}$ [16], evaluate

$$
\overline{\boldsymbol{C}}_{0}^{\mathrm{algD}}{ }_{n+\alpha}=(1-\alpha) \overline{\boldsymbol{C}}_{0}^{\mathrm{algD}}{ }_{n}+\alpha \overline{\boldsymbol{C}}_{0}^{\mathrm{algD}}{ }_{n+1} .
$$

For the inclusion part, the moduli is the Hooke tensor.

7. Applying a similar techniques as in [53], Eq. (30) corresponds to satisfying $\boldsymbol{F}=0$, where $\boldsymbol{F}$ is the stress residual vector. For a time step $\left[t_{n}, t_{n+1}\right]$, where $\Delta \bar{\varepsilon}_{n+1}$ and $\tilde{p}$ are constant, compute the stress residual vector in inclusions as, see Appendix C,

$$
\begin{aligned}
\boldsymbol{F}= & \overline{\boldsymbol{C}}_{0}^{\mathrm{algD}}{ }_{n+\alpha}:\left[\Delta \varepsilon_{\mathrm{I} n+1}-\frac{1}{v_{0}} \boldsymbol{S}^{-1}:\left(\Delta \varepsilon_{\mathrm{I} n+1}-\Delta \overline{\boldsymbol{\varepsilon}}_{n+1}\right)\right] \\
& -\overline{\boldsymbol{C}}_{\mathrm{I}}^{\mathrm{alg}}{ }_{n+\alpha}: \Delta \varepsilon_{\mathrm{I} n+1} .
\end{aligned}
$$

\footnotetext{
${ }^{6}$ In this paper we use $1 / 2$.
} 
8. Check if residual $|\boldsymbol{F}| \leq$ Tol. If so exit the loop.

9. Else, compute Jacobian matrix at constant $\Delta \bar{\varepsilon}_{n+1}$ and $\tilde{p}$, such that $\mathrm{d} \boldsymbol{F}=\boldsymbol{J}: \mathrm{d} \varepsilon_{\mathrm{I}}:$

$$
\begin{aligned}
\boldsymbol{J}= & \frac{\partial \boldsymbol{F}}{\partial \boldsymbol{\varepsilon}_{\mathrm{I}}}+\frac{\partial \boldsymbol{F}}{\partial \boldsymbol{\varepsilon}_{0}}: \frac{\partial \boldsymbol{\varepsilon}_{0}}{\partial \boldsymbol{\varepsilon}_{\mathrm{I}}} \\
= & \overline{\boldsymbol{C}}_{0}^{\mathrm{algD}}{ }_{n+\alpha}:\left[\boldsymbol{I}-\boldsymbol{S}^{-1}\right]-\overline{\boldsymbol{C}}_{\mathrm{I}}^{\mathrm{alg}}{ }_{n+\alpha}-\frac{\partial \overline{\boldsymbol{C}}_{\mathrm{I}}^{\mathrm{alg}}{ }_{n+\alpha}}{\partial \varepsilon_{\mathrm{I}}}: \Delta \boldsymbol{\varepsilon}_{\mathrm{I} n+1}- \\
& \frac{v_{\mathrm{I}}}{v_{0}} \frac{\partial \overline{\boldsymbol{C}}_{0}^{\mathrm{algD}}{ }_{n+\alpha}}{\partial \boldsymbol{\varepsilon}_{0}}:\left[\Delta \boldsymbol{\varepsilon}_{\mathrm{I} n+1}-\boldsymbol{S}^{-1}: \frac{\left(\Delta \boldsymbol{\varepsilon}_{\mathrm{I} n+1}-\Delta \overline{\boldsymbol{\varepsilon}}_{n+1}\right)}{v_{0}}\right]- \\
& \frac{v_{\mathrm{I}}}{v_{0}} \overline{\boldsymbol{C}}_{0}^{\mathrm{algD}}{ }_{n+\alpha}: \boldsymbol{S}^{-1}
\end{aligned}
$$

where

$$
\frac{\partial \overline{\boldsymbol{C}}_{0}^{\mathrm{alg} D}{ }_{n+\alpha}}{\partial \boldsymbol{\varepsilon}_{0}}=(1-D) \frac{\partial \overline{\boldsymbol{C}}_{0}^{\mathrm{alg}}{ }_{n+\alpha}}{\partial \boldsymbol{\varepsilon}_{0}}-\overline{\boldsymbol{C}}_{0}^{\mathrm{alg}}{ }_{n+\alpha} \otimes \frac{\partial D}{\partial \boldsymbol{\varepsilon}_{0}} .
$$

Remark that as Eshelby tensor is evaluated at previous time step, there is no need to compute its derivative.

10. Correct the strain increment in inclusions

$$
\Delta \varepsilon_{\mathrm{I} n+1} \leftarrow \Delta \varepsilon_{\mathrm{I} n+1}+c_{\varepsilon_{\mathrm{I}}} \text { with } \boldsymbol{c}_{\varepsilon_{\mathrm{I}}}=-\boldsymbol{J}^{-1}: \boldsymbol{F},
$$

then start a new iteration (go to step 1).

- After convergence, compute ${ }^{7}$

1. The homogenized stress

$$
\overline{\boldsymbol{\sigma}}_{n+1}=v_{0} \boldsymbol{\sigma}_{0 n+1}+v_{\mathrm{I}} \boldsymbol{\sigma}_{\mathrm{I} n+1} .
$$

2. The linearization of the homogenized stress

$$
\overline{\boldsymbol{C}}_{n+1}^{\mathrm{algD}}=v_{\mathrm{I}} \overline{\boldsymbol{C}}_{\mathrm{I}}^{\mathrm{alg}}{ }_{n+1}: \frac{\partial \boldsymbol{\varepsilon}_{\mathrm{I}}}{\partial \bar{\varepsilon}}+v_{0} \overline{\boldsymbol{C}}_{0}^{\mathrm{algD}}{ }_{n+1}: \frac{\partial \boldsymbol{\varepsilon}_{0}}{\partial \overline{\boldsymbol{\varepsilon}}},
$$

with, see Appendix C,

$$
\begin{aligned}
\frac{\partial \boldsymbol{\varepsilon}_{\mathrm{I}}}{\partial \overline{\boldsymbol{\varepsilon}}} & =-\boldsymbol{J}^{-1}: \frac{\partial \boldsymbol{F}}{\partial \overline{\boldsymbol{\varepsilon}}}, \text { and } \\
\frac{\partial \boldsymbol{\varepsilon}_{0}}{\partial \overline{\boldsymbol{\varepsilon}}} & =\frac{1}{v_{0}}\left(\boldsymbol{I}-v_{1} \frac{\partial \boldsymbol{\varepsilon}_{\mathrm{I}}}{\partial \overline{\boldsymbol{\varepsilon}}}\right) .
\end{aligned}
$$

\footnotetext{
${ }^{7}$ In this formalism, we do not need to evaluate explicitly the strain concentration tensor, nor the macro-moduli, but they follow directly from $\boldsymbol{B}^{\epsilon}=\left\{\boldsymbol{I}+\boldsymbol{S}:\left[\left(\overline{\boldsymbol{C}}_{0}^{\text {algD }}{ }_{n+\alpha}\right)^{-1}: \overline{\boldsymbol{C}}_{\mathrm{I}}^{\text {alg }}{ }_{n+\alpha}-\right.\right.$ $\boldsymbol{I}]\}^{-1}$, and $\overline{\boldsymbol{C}}_{n+\alpha}=\left[v_{\mathrm{I}} \overline{\boldsymbol{C}}_{\mathrm{I}}^{\text {alg }}{ }_{n+\alpha}: \boldsymbol{B}^{\epsilon}+v_{0} \overline{\boldsymbol{C}}_{0}^{\text {algD }}{ }_{n+\alpha}\right]:\left[v_{1} \boldsymbol{B}^{\epsilon}+v_{0} \boldsymbol{I}\right]^{-1}$, respectively.
} 
3. With a view toward the evaluation of the Jacobian matrix of the coupled system, the linearization of the homogenized stress with respect to $\tilde{p}$

$$
\boldsymbol{C}_{n+1}^{\tilde{p}}=v_{\mathrm{I}} \overline{\boldsymbol{C}}_{\mathrm{I}}^{\mathrm{alg}}: \frac{\partial \boldsymbol{\varepsilon}_{\mathrm{I}}}{\partial \tilde{p}}+v_{0} \overline{\boldsymbol{C}}_{0}^{\mathrm{algD}}{ }_{n+1}: \frac{\partial \boldsymbol{\varepsilon}_{0}}{\partial \tilde{p}},
$$

with

$$
\begin{aligned}
\frac{\partial \varepsilon_{\mathrm{I}}}{\partial \tilde{p}} & =-\boldsymbol{J}^{-1}: \frac{\partial \boldsymbol{F}}{\partial \tilde{p}}, \text { and } \\
\frac{\partial \boldsymbol{\varepsilon}_{0}}{\partial \tilde{p}} & =-\frac{v_{1}}{v_{0}} \frac{\partial \boldsymbol{\varepsilon}_{\mathrm{I}}}{\partial \tilde{p}} .
\end{aligned}
$$

At the end of the M-T process, the effective stress $\bar{\sigma}$ is known from Eq. (39). In order to solve the macro-scale problem, as exposed in section 3.2, the Jacobian matrix of the fully coupled problem (including the unknown $\tilde{p}$ ) has to be evaluated.

\subsubsection{Linearization of the homogenized fields}

At the end of the M-T process, $\overline{\boldsymbol{\sigma}}$ is obtained from Eq. (39), $\overline{\boldsymbol{C}}^{\mathrm{algD}}$, its derivative with respect to $\bar{\varepsilon}$ at constant damage, from Eq. (40), and $\overline{\boldsymbol{C}}^{\tilde{p}}$, its derivative with respect to $\tilde{p}$ at constant deformation, from Eq. (43). Thus, the linearization (27), after having performed the M-T process, of the fully coupled problem reads

$$
\begin{aligned}
\delta \overline{\boldsymbol{\sigma}} & =\left[\overline{\boldsymbol{C}}^{\mathrm{algD}}-v_{0}\left(\hat{\boldsymbol{\sigma}}_{0} \otimes \frac{\partial D}{\partial \boldsymbol{\varepsilon}_{0}}\right): \frac{\partial \boldsymbol{\varepsilon}_{0}}{\partial \overline{\boldsymbol{\varepsilon}}}\right]: \delta \overline{\boldsymbol{\varepsilon}} \\
& +\left[\boldsymbol{C}^{\tilde{p}}-v_{0}\left(\hat{\boldsymbol{\sigma}}_{0} \otimes \frac{\partial D}{\partial \boldsymbol{\varepsilon}_{0}}\right): \frac{\partial \varepsilon_{0}}{\partial \tilde{p}}-v_{0} \hat{\boldsymbol{\sigma}}_{0} \frac{\partial D}{\partial \tilde{p}}\right] \delta \tilde{p},
\end{aligned}
$$

where $\frac{\partial \varepsilon_{0}}{\partial \bar{\varepsilon}}$ and $\frac{\partial \varepsilon_{0}}{\partial \tilde{p}}$ are obtained from (42) and (45) respectively.

To fully characterize the micro-mechanics in the non-local framework, the representation of the matrix accumulated plastic strain on the RVE is linearized in terms of $\delta \bar{\varepsilon}$ and $\delta \tilde{p}$. Using the elasto-plastic formalism presented in section 2.2.4, but applied on the homogenized matrix effective stress $\hat{\sigma}_{0}$, the accumulated plastic strain variation of the matrix can be calculated in the effective stress space following the same argument as in [54, chapter 12]. Eventually, one has

$$
\delta p=\frac{1}{h} \boldsymbol{N}: \overline{\boldsymbol{C}}_{0}^{\mathrm{el}}:\left[\delta \varepsilon_{0}-(\triangle p) \frac{\partial \boldsymbol{N}}{\partial \hat{\boldsymbol{\sigma}}_{0}}: \delta \hat{\boldsymbol{\sigma}}_{0}\right],
$$

where $h=3 \mu_{0}+\frac{\mathrm{d} R}{\mathrm{~d} p}$. For $J_{2}$-plasticity,

$$
\overline{\boldsymbol{C}}_{0}^{\mathrm{el}}: \frac{\partial \boldsymbol{N}}{\partial \hat{\boldsymbol{\sigma}}_{0}}=2 \mu_{0} \frac{\partial \boldsymbol{N}}{\partial \hat{\boldsymbol{\sigma}}_{0}},
$$


where $\mu_{0}$ is the shear modulus of the matrix material. Since $\boldsymbol{N}: \frac{\partial \boldsymbol{N}}{\partial \hat{\boldsymbol{\sigma}}_{0}}=0$ and $\boldsymbol{N}: \overline{\boldsymbol{C}}_{0}^{\text {el }}=2 \mu_{0} \boldsymbol{N}$ for isotropic materials, Eq. (47) can be rewritten

$$
\delta p=\frac{1}{h} \boldsymbol{N}: \overline{\boldsymbol{C}}_{0}^{\mathrm{el}}: \delta \boldsymbol{\varepsilon}_{0}=\frac{2 \mu_{0}}{h} \boldsymbol{N}:\left(\frac{\partial \varepsilon_{0}}{\partial \overline{\boldsymbol{\varepsilon}}}: \delta \overline{\boldsymbol{\varepsilon}}+\frac{\partial \varepsilon_{0}}{\partial \tilde{p}} \delta \tilde{p}\right),
$$

where $\boldsymbol{N}=\frac{\partial f}{\partial \hat{\boldsymbol{\sigma}}_{0}^{\text {tr }}}$, and where $\hat{\boldsymbol{\sigma}}_{0}^{\mathrm{tr}}$ is the trial (elastic predictor) tensor of the effective stress in the matrix.

Note that the values $p$ and $N$ are representations of the local equivalent plastic strain and of the yield normal in the matrix obtained during the MFH process. They do not correspond to average values in the matrix phase.

Now that the RVE is characterized by the linearization $\delta \overline{\boldsymbol{\sigma}}$ of the RVE homogenized stress tensor and by the linearization $\delta p$ of the RVE representation of the accumulated plastic strain in the matrix, the macro-scale formulation can be defined in terms of the homogenized properties.

\subsection{The gradient-enhanced damage model in the macro-scale analysis}

At the macro-scale, a finite element formulation, based on the weak form of the governing equations, is implemented. This finite element formulation accounts for a new degree of freedom on each node, which results from the resolution of the new Helmholtz-type equations governing the implicit formulation of the gradient-enhanced damage model. In this section, the governing non-linear equations are recalled and expressed in a weak form before being linearized to introduce a Newton-Raphson resolution.

\subsubsection{Governing equations}

The problem is limited to small deformations and static analysis. The governing equations of the homogenized material using the implicit gradient enhanced elasto-plasticity read

$$
\begin{array}{cl}
\nabla \cdot \boldsymbol{\sigma}+\boldsymbol{f}=\boldsymbol{0} & \text { for the material, } \\
\tilde{p}-l^{2} \nabla^{2} \tilde{p}=p & \text { for the matrix material only, }
\end{array}
$$

where $\boldsymbol{f}$ represents the body force vector, and $\tilde{p}$ is a homogenized representation of the non-local accumulated plastic strain of the matrix material. Standard boundary conditions -Neumann and/or Dirichlet- are applied to the first set of partial differential equations (PDE) (50). For the implicit gradient formulation, according to section 2.2.3, homogeneous Neumann boundary conditions are applied:

$$
\frac{\partial \tilde{p}}{\partial n}=n_{i} \frac{\partial \tilde{p}}{\partial x_{i}}=0 \quad \text { on } \quad \Gamma
$$

where $n$ is the unit outward normal to the external boundary $\Gamma$ of the body ${ }^{8}$. This equation expresses "that no direct energy exchange can exist with the

${ }^{8}$ The discussion on the boundary definition for direct numerical simulations of unit cells is reported in section 4.1 
surroundings of the body and ensures that the net dissipation in the body vanishes for constant damage" [55]

However, as it will be shown in the next section, this boundary condition will be accounted for when establishing the finite-element formulation, and will not have to be enforced explicitly during the computation.

\subsubsection{Weak formulation}

Weak form of the set of Eqs. (50-51) is established using suitable weight functions defined in the $n+1$ - dimensional spaces:

$\boldsymbol{w}_{u} \in\left[C^{0}\right]^{n} \quad$ The weight function of the displacement field,

$w_{\tilde{p}} \in\left[C^{0}\right] \quad$ The weight function of non-local accumulated plastic strain of the matrix material.

Multiplying equilibrium condition (50) with the displacement weight function $\boldsymbol{w}_{u}$ and integrating the result over the domain $\Omega$ yields

$$
\int_{\Omega} \boldsymbol{w}_{u} \cdot[\nabla \cdot \boldsymbol{\sigma}+\boldsymbol{f}] \mathrm{d} \Omega=0 .
$$

Applying the divergence theorem, the natural boundary conditions for the boundary traction vector $(\boldsymbol{\sigma} \cdot \boldsymbol{n}=\boldsymbol{T})$ on $\Gamma_{T}$, the essential boundary conditions on $\Gamma-\Gamma_{T}$ and the symmetry property of the stress tensor leads to

$$
\int_{\Omega}\left[\nabla \boldsymbol{w}_{u}\right]^{\mathrm{T}}: \boldsymbol{\sigma} \mathrm{d} \Omega=\int_{\Omega} \boldsymbol{w}_{u} \cdot \boldsymbol{f} \mathrm{d} \Omega+\int_{\Gamma_{T}} \boldsymbol{w}_{u} \cdot \boldsymbol{T} \mathrm{d} \Gamma .
$$

The same method is used to treat the second PDE (51), which results in:

$$
\int_{\Omega}\left(w_{\tilde{p}} \tilde{p}+l^{2} \nabla w_{\tilde{p}} \cdot \nabla \tilde{p}\right) \mathrm{d} \Omega=\int_{\Omega} w_{\tilde{p}} p \mathrm{~d} \Omega,
$$

where (52) has been used. Note that as the boundary conditions (52) have already been considered herein, they will not have to be explicitly applied in the finite element formulation.

\subsubsection{Linearization}

Considering a time step $\left[t_{n}, t_{n+1}\right]$, it is assumed that the non-linear system (54-55) has been solved at time $t_{n}$. The problem is stated as solving the following equations at time $t_{n+1}$ :

$$
\begin{array}{r}
\int_{\Omega}\left[\nabla \boldsymbol{w}_{u}\right]^{\mathrm{T}}: \boldsymbol{\sigma}_{n+1} \mathrm{~d} \Omega=\int_{\Omega} \boldsymbol{w}_{u} \cdot \boldsymbol{f}_{n+1} \mathrm{~d} \Omega+\int_{\Gamma_{T}} \boldsymbol{w}_{u} \cdot \boldsymbol{T}_{n+1} \mathrm{~d} \Gamma, \\
\int_{\Omega}\left(w_{\tilde{p}} \tilde{p}_{n+1}+l^{2} \nabla w_{\tilde{p}} \cdot \nabla \tilde{p}_{n+1}\right) \mathrm{d} \Omega=\int_{\Omega} w_{\tilde{p}} p_{n+1} \mathrm{~d} \Omega .
\end{array}
$$

The set of non-linear equations (56-57) can be recast in the following form:

$$
\left.\Psi(\sigma, \tilde{p})\right|_{t_{n+1}}=0,
$$


which is solved using the Newton-Raphson method. Thus, at each iteration $i$ of time step $\left[t_{n}, t_{n+1}\right]$, we have

$$
\Psi\left(\boldsymbol{\sigma}_{n+1}^{i}, \tilde{p}_{n+1}^{i}\right)+\left.\frac{\partial \Psi}{\partial(\boldsymbol{\sigma}, \tilde{p})}\right|_{\boldsymbol{\sigma}_{n+1}^{i}, \tilde{p}_{n+1}^{i}}\left[\left(\boldsymbol{\sigma}_{n+1}^{i+1}, \tilde{p}_{n+1}^{i+1}\right)-\left(\boldsymbol{\sigma}_{n+1}^{i}, \tilde{p}_{n+1}^{i}\right)\right]=0 .
$$

Using Eqs. (56- 57), the explicit form of Eq. (59) can be expressed as

$$
\begin{aligned}
\int_{\Omega}\left[\nabla \boldsymbol{w}_{u}\right]^{\mathrm{T}}: & \delta \boldsymbol{\sigma}_{n+1}^{i+1} \mathrm{~d} \Omega= \\
& \int_{\Omega} \boldsymbol{w}_{u} \cdot \boldsymbol{f}_{n+1} \mathrm{~d} \Omega+\int_{\Gamma_{T}} \boldsymbol{w}_{u} \cdot \boldsymbol{T}_{n+1} \mathrm{~d} \Gamma-\int_{\Omega}\left[\nabla \boldsymbol{w}_{u}\right]^{\mathrm{T}}: \boldsymbol{\sigma}_{n+1}^{i} \mathrm{~d} \Omega,
\end{aligned}
$$

and

$$
\begin{aligned}
-\int_{\Omega} w_{\tilde{p}} \delta p_{n+1}^{i+1} \mathrm{~d} \Omega+ & \int_{\Omega}\left[w_{\tilde{p}} \delta \tilde{p}_{n+1}^{i+1}+l^{2} \nabla w_{\tilde{p}} \cdot \nabla\left(\delta \tilde{p}_{n+1}^{i+1}\right)\right] \mathrm{d} \Omega= \\
& \int_{\Omega} w_{\tilde{p}} p_{n+1}^{i} \mathrm{~d} \Omega-\int_{\Omega}\left[w_{\tilde{p}} \tilde{p}_{n+1}^{i}+l^{2} \nabla w_{\tilde{p}} \cdot \nabla \tilde{p}_{n+1}^{i}\right] \mathrm{d} \Omega .
\end{aligned}
$$

According to the classical resolution schemes, the key issue of solving the weak form (60-61) is to find the expressions of $\delta \boldsymbol{\sigma}_{n+1}^{i+1}$ and $\delta p_{n+1}^{i+1}$ in terms of $\delta \varepsilon_{n+1}^{i+1}$ and $\delta \tilde{p}_{n+1}^{i+1}$, which results from Eqs. (46) and (49). The resolution can now be achieved using a classical finite-element (FE) formulation.

\subsection{Finite element implementation - Discretization and incremental-iterative formulation}

After substituting Eqs. (46) and (49) into the linearized weak form (6061 ), the finite element discretization is straightforwardly formulated using the Galerkin approach. Toward this end, the displacement field $U$ and the non-local accumulated plastic strain field $\tilde{p}$ can be interpolated in each element $\Omega_{\mathrm{e}}$ using traditional shape function matrices $\boldsymbol{N}_{u}$ and $\boldsymbol{N}_{\tilde{p}}$ as follows:

$$
\boldsymbol{U}=\boldsymbol{N}_{u} \boldsymbol{u}, \quad \text { and } \quad \tilde{p}=\boldsymbol{N}_{\tilde{p}} \tilde{\boldsymbol{p}},
$$

where the vectors $\boldsymbol{u}$ and $\tilde{\boldsymbol{p}}$ contain the assembled nodal values of the displacements and of the non-local accumulated plastic strain field, respectively. Similarly, the weight functions are interpolated using the same shape functions

$$
\boldsymbol{w}_{u}=\boldsymbol{N}_{u} \boldsymbol{d} \boldsymbol{u}, \quad \text { and } \quad w_{\tilde{p}}=\boldsymbol{N}_{\tilde{p}} \boldsymbol{d} \tilde{\boldsymbol{p}},
$$

where $\boldsymbol{d} \boldsymbol{u}$ and $\boldsymbol{d} \tilde{\boldsymbol{p}}$ are arbitrary values fulfilling the essential boundary conditions.

The macro-strain tensorial field and the gradient field of the non-local accumulated plastic strain can easily be deduced, in terms of the problem unknowns, from

$$
\overline{\boldsymbol{\varepsilon}}=\boldsymbol{B}_{u} \boldsymbol{u}, \quad \text { and } \quad \nabla \tilde{p}=\nabla \boldsymbol{N}_{\tilde{p}} \tilde{\boldsymbol{p}}=\boldsymbol{B}_{\tilde{p}} \tilde{\boldsymbol{p}}
$$


where $\boldsymbol{B}_{u}$ and $\boldsymbol{B}_{\tilde{p}}$ represent the matrix strain operator and the matrix operator for the non-local accumulated plastic strain, respectively. Therefore, using Eq. (63), and the arbitrary nature of $\boldsymbol{d} \boldsymbol{u}$ and $\boldsymbol{d} \tilde{\boldsymbol{p}}$, the linearized weak form (60-61) can be stated in the following matrix form at iteration ' $i$ ' of time step $\left[t_{n}, t_{n+1}\right]$ :

$$
\left[\begin{array}{ll}
\boldsymbol{K}_{u u}^{i} & \boldsymbol{K}_{u \tilde{p}}^{i} \\
\boldsymbol{K}_{\tilde{p} u}^{i} & \boldsymbol{K}_{\tilde{p} \tilde{p}}^{i}
\end{array}\right]\left[\begin{array}{c}
\delta \boldsymbol{u} \\
\delta \tilde{p}
\end{array}\right]=\left[\begin{array}{c}
\boldsymbol{F}_{\mathrm{ext}}^{n+1}-\boldsymbol{F}_{\mathrm{int}}^{i} \\
\boldsymbol{F}_{p}^{i}-\boldsymbol{F}_{\tilde{p}}^{i}
\end{array}\right]
$$

with the sub-matrices defined by

$$
\begin{aligned}
\boldsymbol{K}_{u u}^{i} & =\int_{\Omega} \boldsymbol{B}_{u}^{\mathrm{T}} \overline{\boldsymbol{C}}_{\mathrm{D}}^{i} \boldsymbol{B}_{u} \mathrm{~d} \Omega, \\
\boldsymbol{K}_{u \tilde{p}}^{i} & =\int_{\Omega} \boldsymbol{B}_{u}^{\mathrm{T}} \boldsymbol{S}_{\boldsymbol{D}}{ }^{i} \boldsymbol{N}_{\tilde{p}} \mathrm{~d} \Omega, \\
\boldsymbol{K}_{\tilde{p} u}^{i} & =-\int_{\Omega} \boldsymbol{N}_{\tilde{p}}^{\mathrm{T}} \boldsymbol{S}_{\boldsymbol{p}}{ }^{i} \boldsymbol{B}_{u} \mathrm{~d} \Omega, \text { and } \\
\boldsymbol{K}_{\tilde{p} \tilde{p}}^{i} & =\int_{\Omega}\left[\left(1-S_{\tilde{p}}^{i}\right) \boldsymbol{N}_{\tilde{p}}^{\mathrm{T}} \boldsymbol{N}_{\tilde{p}}+l^{2} \boldsymbol{B}_{\tilde{p}}^{\mathrm{T}} \boldsymbol{B}_{\tilde{p}}\right] \mathrm{d} \Omega .
\end{aligned}
$$

In these last expressions, $\overline{\boldsymbol{C}}_{\mathrm{D}}^{i}, \boldsymbol{S}_{\boldsymbol{D}}{ }^{i}, \boldsymbol{S}_{\boldsymbol{p}}{ }^{i}$ and $S_{\tilde{p}}^{i}$ are the matrix representations [54, appendix C] of the previously defined tensors $\left[\overline{\boldsymbol{C}}^{\mathrm{algD}}-v_{0}\left(\hat{\boldsymbol{\sigma}}_{0} \otimes \frac{\partial D}{\partial \boldsymbol{\varepsilon}_{0}}\right)\right.$ : $\left.\frac{\partial \boldsymbol{\varepsilon}_{0}}{\partial \overline{\boldsymbol{\varepsilon}}}\right]\left.\right|_{i},\left.\quad\left[\boldsymbol{C}^{\tilde{p}}-v_{0}\left(\hat{\boldsymbol{\sigma}}_{0} \otimes \frac{\partial D}{\partial \boldsymbol{\varepsilon}_{0}}\right): \frac{\partial \boldsymbol{\varepsilon}_{0}}{\partial \tilde{p}}-v_{0} \hat{\boldsymbol{\sigma}}_{0} \frac{\partial D}{\partial \tilde{p}}\right]\right|_{i}, \frac{2 \mu_{0}}{h^{i}} \boldsymbol{N}^{i}:\left.\frac{\partial \boldsymbol{\varepsilon}_{0}}{\partial \overline{\boldsymbol{\varepsilon}}}\right|_{i}$ and $\frac{2 \mu_{0}}{h^{i}} \boldsymbol{N}^{i}:$ $\left.\frac{\partial \varepsilon_{0}}{\partial \tilde{p}}\right|_{i}$ respectively. Finally the force vectors are easily obtained from

$$
\begin{aligned}
\boldsymbol{F}_{\mathrm{ext}}^{n+1} & =\int_{\Omega} \boldsymbol{N}_{u}^{\mathrm{T}} \boldsymbol{f}_{n+1} \mathrm{~d} \Omega+\int_{\Gamma_{T}} \boldsymbol{N}_{u}^{\mathrm{T}} \boldsymbol{T}_{n+1} \mathrm{~d} \Gamma \\
\boldsymbol{F}_{\mathrm{int}}^{i} & =\int_{\Omega} \boldsymbol{B}_{u}^{\mathrm{T}} \overline{\boldsymbol{\sigma}}^{i} \mathrm{~d} \Omega, \\
\boldsymbol{F}_{p}^{i} & =\int_{\Omega} \boldsymbol{N}_{\tilde{p}}^{\mathrm{T}} \boldsymbol{p}^{i} \mathrm{~d} \Omega, \text { and } \\
\boldsymbol{F}_{\tilde{p}}^{i} & =\int_{\Omega}\left(\boldsymbol{N}_{\tilde{p}}^{\mathrm{T}} \boldsymbol{N}_{\tilde{p}}+l^{2} \boldsymbol{B}_{\tilde{p}}^{\mathrm{T}} \boldsymbol{B}_{\tilde{p}}\right) \tilde{\boldsymbol{p}}^{i} \mathrm{~d} \Omega .
\end{aligned}
$$

\section{Numerical simulations}

The new gradient enhanced MFH model described in section 3 has been implemented as a non-linear FE module in GMSH [56]. Two kinds of lockingfree elements have been used. On the one hand linear hexahedra with selected reduced integration, and on the other hand, quadratic tetrahedra with 4 Gauss points. The same polynomial order is used for the different fields. Although the method could also be used with 2D elements, only the most general 3D formulation is implemented. The numerical verification of the model is first carried out by comparing the results obtained from the homogenized law to the results obtained from direct numerical simulations on unit cells for different fibers 
percentages. As the MFH process implemented uses the non-local Lemaitre Chaboche ductile damage model described in section 2.2.4, this non-local model is equally available for the direct numerical simulations of the composite. Afterward, the gradient enhanced MFH model is applied to simulate the response of a notched sample under transverse loading.

\subsection{Numerical verifications}

In this section, the gradient enhanced MFH model is used to compute the effective response of unidirectional fiber reinforced elasto-plastic composites. The considered composites consist in a continuous elasto-plastic matrix, experiencing damage, reinforced by unidirectional continuous fibers with linear elastic behavior. As an example, the matrix considered is made of epoxy and the reinforcements are continuous carbon fibers.

\subsubsection{Tests description}

The properties of the matrix are: Young's modulus $E_{0}=2.89 \mathrm{GPa}$, Poisson's ratio $\nu_{0}=0.3$ and yielding stress $\sigma_{Y}=35.0 \mathrm{MPa}$. The hardening law of the epoxy material follows an exponential law

$$
R(p)=h_{0}\left[1-e^{-m p}\right],
$$

where $h_{0}=73.0 \mathrm{MPa}$ and $m=60$. The damage law obeys Eq. (20), with the parameters $S_{0}=2.0 \mathrm{MPa}, s=0.5$ and $p_{C}=0.0$, which are reference values for the unidirectional carbon fiber reinforced epoxy [46]. By lack of data for epoxy matrix, we consider these composite damage values for the matrix.

The carbon fibers are linear elastic, with $E_{\mathrm{I}}=238 \mathrm{GPa}$ and $\nu_{\mathrm{I}}=0.26$. Moreover, the carbon fibers are assumed to be isotropic, which is far from being true $^{9}$, to enhance the difference of material properties in the transverse direction and, thus, to illustrate the ability of the method to perform the homogenization. Results obtained will thus not match experimental results but will allow to verify the new MFH process developed.

Reference results are provided by finite element computations performed on unit cells where the micro-structure is fully meshed. In these unit cells, it is assumed that the matrix and the fibers can be considered as homogeneous materials. As these simulations involve softening, the representativeness of the unit cells is disputable as a shear band of comparable size of the volume appears [33, 34, 57-59]. This requires particular attention, as using averaging methods [59], proscribing periodic boundary conditions but applying adequate boundary conditions [33, 34], when extracting the macroscopic behavior from the cell simulations, particularly when performing a computational homogenization. However, such methods are beyond the scope of this paper whose main purpose is the development of a MFH method, for which the RVE remains conceptual.

With the non-local model, the Helmholtz equation (51) has to be solved in the domain of interest with the associate boundary conditions (52) on its

${ }^{9}$ The Young modulus of fibers in transverse direction is lower than in longitudinal direction. 


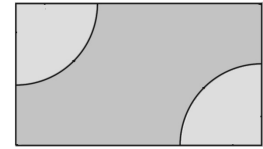

(a) Periodic unit cell

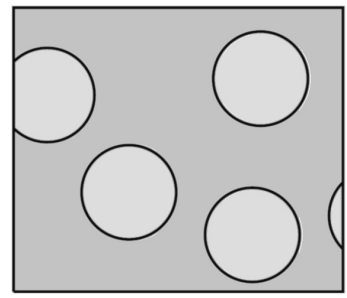

(b) Random unit cell

Figure 2: The transverse section (the meshes are actually 3D) of the two kinds of studied unit cells for unidirectional continuous fiber reinforced composite with a volume fraction $v_{\mathrm{I}}=0.3$.

boundary. When using the MFH, the domain boundary is the boundary of the domain under consideration. However, when considering unit cells made of dissipative and elastic materials arises the question on how to apply the boundary conditions (52). A complete discussion on the thermodynamical aspect of the method can be found in [55], and can be summarized as

- The boundary condition A (BC A) corresponds to applying the boundary conditions $(52)$ to the boundary of the unit cells and integrating the Helmholtz equation (51) in the two material components. As one material remains elastic, the non-local quantity is thus also calculated by implicit averaging over the elastic inclusions. Although not thermodynamically rigorous, "this limit can be interpreted as gradually switching off any non-local effects in the elastic regime, while preserving the ability of the elastic region to influence damage growth in the process zone" [55].

- The boundary condition B (BC B) corresponds to applying the boundary conditions (52) at the interface between the two materials, restricting direct energy exchanges to the process zone. From the analyzes in [55] this option may appear as too limiting as "it seems unlikely that energy exchange would be stopped at the boundary of the process zone".

- The boundary condition $\mathrm{C}(\mathrm{BC} \mathrm{C})$ corresponds to balancing the energy exchange across the interfaces.

As a complete study of this effect is out of the scope of this paper, when performing the numerical simulations of the unit cell, we will consider the different results obtained for BCs A and B only.

In this paper, two kinds of unit cells are applied for the validation. The first one is the small unit cell with an hexagonal periodic distribution of fibers in the transverse direction (Fig.2(a)), and the second one is the larger cell with random distribution of fibers in the transverse direction (Fig. 2(b)). It is assumed that the cross section of the fibers is circular with a radius of $50 \mu \mathrm{m}$. Then, the 
size of the unit cell can be calculated according to the fiber volume fraction $v_{\text {I }}$ and the number of fibers considered in the simulation [60]. In the second case, as the size of the unit cell is not much larger than the micro-structure length (only four fibers per cell are considered), six different occurrences, with random fiber distributions are simultaneously considered in order to capture the scattering effect due to the violation of the length scale separation. As we choose traction-free/displacement-controlled boundary conditions, the cell with only four fibers allows obtaining results close to the periodic cell results, as shown in the following part. The periodic cells have about 250 linear hexahedral elements, while the non-periodic unit cells have about 2500 quadratic tetrahedral elements, which allows to reach mesh convergence ${ }^{10}$. All the meshes have one layer of elements on the thickness. The comparisons between simulations are successively achieved for 15,30 and $50 \%$ volume fractions of fibers. For the periodic cells, BCs A and B are considered, while for the random distribution cells, only the BCs A are considered.

The characteristic length of the non-local model in the simulations of unit cells should be related to the width of the shear band in the matrix material. The size of the unit cells is about a few hundreds micro-meters, which is much smaller than the characteristic length of the matrix material, and the interactions between the matrix material points are blocked off by the elastic fibers. Therefore, the simulations on unit cells are not sensitive to reasonable values of $l$. As only a simple tensile test is considered, the MFH simulation will give uniform numerical results, which are not affected by the value of $l$. So we set $l^{2}=2.0 \mathrm{~mm}^{2}$, which is a typical value for composite [49].

\subsubsection{Results}

First, a loading-unloading cycle is applied in the direction transverse to the fibers. A maximal deformation of $10 \%$ is reached before the unloading proceeds to zero-transverse deformation. Boundary conditions correspond to uniform displacements ${ }^{11}$, with controlled deformation in the loading direction, traction-free condition in the other transverse direction and plane-strain condition in the longitudinal direction. The plane-strain condition in the fibers direction correspond to the state of a composite ply, while the combination of traction-free, strain-controlled displacements in the transverse directions gives good convergence results in terms of unit-cell size.

The effective behaviors predicted by the MFH models and the FE results for the three fiber volume ratios are presented in Fig. 3. For the three fiber volume ratios, the homogenized property is dominated by the properties of the matrix, with an obvious elasto-plastic behavior exhibiting softening. When considering the periodic cells, BC B predicts higher softening than $\mathrm{BC}$ A, as it

\footnotetext{
${ }^{10}$ Number of elements is not exactly the same for all the fiber volume fractions nor for all the realizations of the unit cells

${ }^{11}$ For the periodic unit cells and the MFH problems, this corresponds to periodic boundary conditions by symmetry.
} 


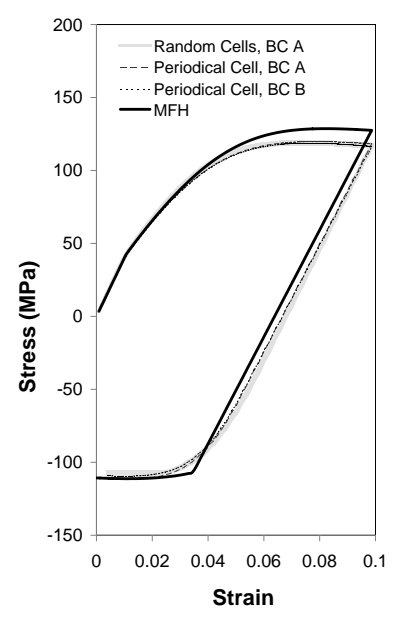

(a) $v_{\mathrm{I}}=0.15$

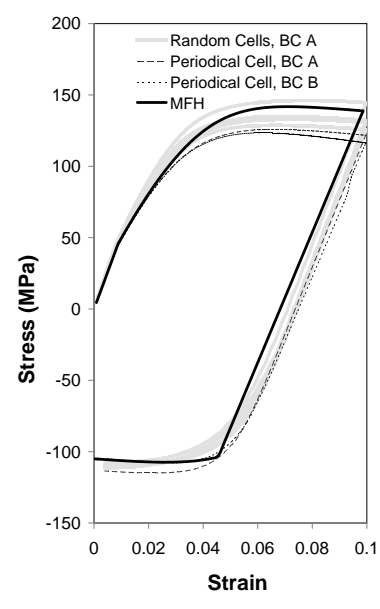

(b) $v_{\mathrm{I}}=0.30$

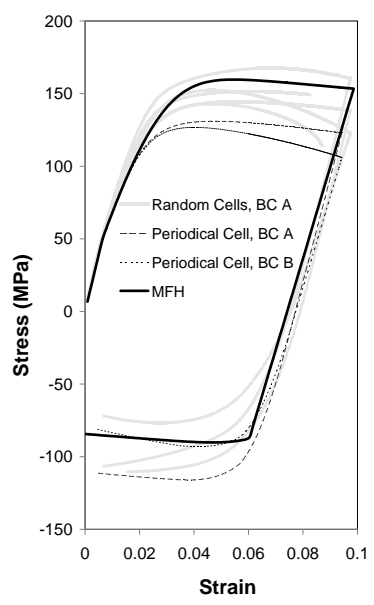

(c) $v_{\mathrm{I}}=0.50$

Figure 3: Stress-strain behavior of the epoxy fiber reinforced composite, for different fiber volume ratios, under transverse loading.

was expected since matrix constraints are lower. However, for low to moderate (up to $30 \%$ fiber volume ratio), the difference remains reduced. The 6 random cell occurrences converged as they exhibit a solution similar to the periodic unit cell, although the statistical dispersion increases with the fiber volume ratio. For the fiber volume fractions of $v_{\mathrm{I}}=15 \%$ and $30 \%$, rather good predictions are given by the MFH model, with, as expected, higher macroscopic stress and damage predicted by the MFH due to the incremental LCC formulation. However for $v_{\mathrm{I}}=50 \%$, the MFH model overestimates the macroscopic stress compared to the periodic unit cell results. This error comes from the assumption of Mori - Tanaka based MFH, which can give an accurate prediction when the volume fraction of inclusions is lower than $30 \%$. Also, for the computations of random cells of $v_{\mathrm{I}}=50 \%$, only three random cells reach the end of the loadingunloading cycle. This might be due to the abrupt increase of damage, which can reach 1.0 in high stress concentration area before the maximum loading stage is reached. More explanations for this will be given in the next section. Note that these curves are for comparison purpose as few composites will sustain this level of deformation.

For illustration purpose, the von Mises stress, the matrix accumulated plastic strain and the matrix damage for one $30 \%$ fiber random cell are presented in Fig. 4 at zero-strain after unloading. As comparison, those values obtained from the MFH are also presented. In the random unit cell, the distribution of the von Mises stress is quite homogeneous (Fig. 4(a)), higher damage is found between the fibers along the loading direction (Fig. 4(c)), and higher accumulated plastic strain appears in a $45^{\circ}$-band (Fig. 4(b)). Highest values of those in matrix are seen at the fiber/matrix interfaces. On the contrary, 


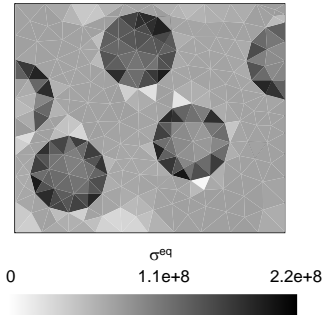

(a) Von Mises stress $(\mathrm{Pa})$

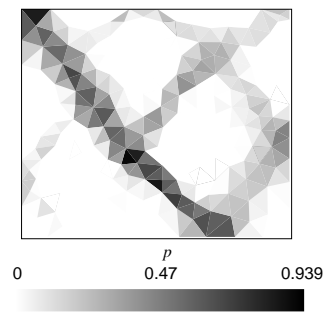

(b) Local accumulated plastic strain

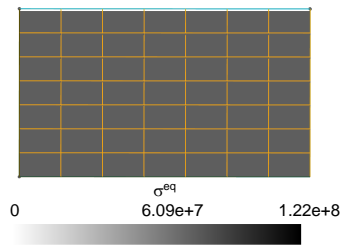

(d) Von Mises stress (Pa)

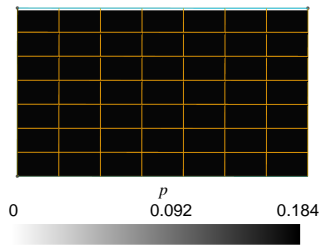

(e) Local accumulated plastic strain

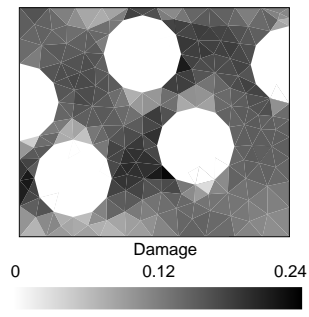

(c) Damage

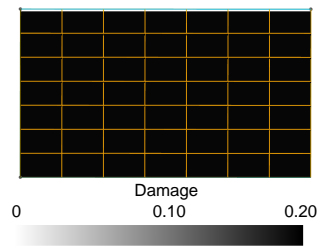

(f) Damage

Figure 4: The von Mises stress, accumulated plastic strain in the matrix phase and damage in the matrix phase at zero-strain after unloading and plastic compression, for a $30 \%$ fibers random unit cell (a) (c), and those of a 30\% fiber homogenized cell (d) (f) under transverse loading. The scale for the von Mises stress shows the maximal value reached during the loading process.

uniform values of the von Mises stress, accumulated plastic strain and damage are obtained by the cell with the MFH process (Fig. 4(d)-Fig. 4(f)). This was expected, but as the homogenized material exhibits softening, it is important to make sure localization problems are avoided under uniform loading, which is the case in this formulation.

The constant non-local damage in the matrix obtained with the MFH scheme is compared, in Fig. 5, to the minimum, average, and maximal values reached in the epoxy matrix for the periodic cell simulations, for $30 \%$ fiber volume fraction. For the periodic cells, the effect of the boundary conditions considered for the Helmholtz equation is studied. From this figure, it can be seen that the damage in the periodic cell has a higher average value when applying $\mathrm{BC}$ $\mathrm{B}$, as expected. The non-local $\mathrm{MFH}$ damage remains in-between the average and (minimal) maximal value of the matrix, for $\mathrm{BC} A(\mathrm{~B})$, respectively. This confirms that the damage predicted by the $\mathrm{MFH}$ is a good image of the average value in the matrix.

Next, loading along the fiber direction is studied. Boundary conditions correspond to uniform displacements, with controlled deformation in the loading direction, traction-free and plane-strain conditions in the two transverse directions. The effective behaviors predicted by the MFH models and the FE results 


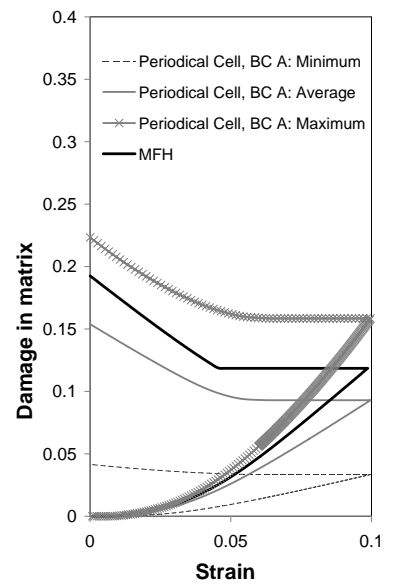

(a) $\mathrm{BC} \mathrm{A}$

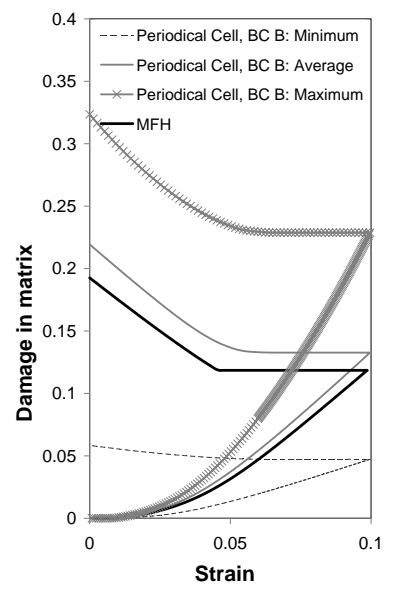

(b) $\mathrm{BC} \mathrm{B}$

Figure 5: Damage evolutions in the epoxy matrix for the $30 \%$ fiber periodic cell and for the $30 \%$ fiber homogenized cell. Results obtained with the MFH scheme are compared to the minimum, average and maximum values reached for the periodic cell simulations, and for $\mathrm{BC}$ $\mathrm{A}$ and $\mathrm{BC} \mathrm{B}$ related to the non-local Helmholtz equation.

for the three fiber volume ratios are presented in Fig.6. As continuous fibers are considered in our case, only the periodic unit cells, with BC A, are applied to obtain the reference results, as random cells give the same results. For all the three fiber volume fractions, nearly linear elastic properties have been obtained in this longitudinal direction. This results from the large difference between the Young's modulus of the matrix and that of the fibers, which makes the homogenized property dominated by the fibers. From Fig. 6, it is found that the MFH model underestimates the macroscopic stress within $5 \sim 10 \%$ difference, while it was found that the MFH model overestimates the macroscopic stress in the transverse direction.

\subsection{Structural application}

In this section, a two-dimensional example is studied using the gradient enhanced MFH formulation presented previously, and in particular the convergence of the proposed formulation during the strain softening stage is investigated. The same material system as in section 4.1 is considered herein, with the characteristic length $l^{2}=2.0 \mathrm{~mm}^{2}$. It should be noted that this value is just an assumption for this simulation. Indeed, for a real material, on the one hand fibers have the effect of preventing the interactions among the matrix material points in transverse direction, and on the other hand, they dominate the long range effects in the longitudinal direction [61]. So different values of $l$, which are related to the size of fibers and internal distances among fibers, should be used in the different material directions, motivating the development of an anisotropic non-local model. Moreover, an artificial spreading of the damaged 


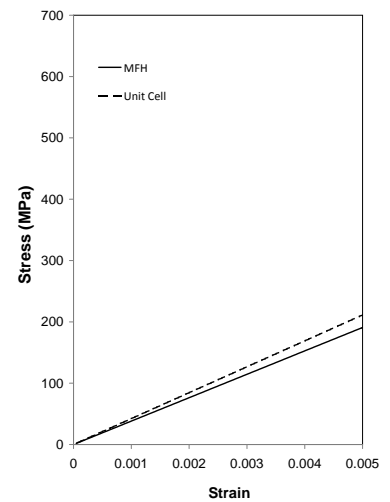

(a) $v_{\mathrm{I}}=0.15$

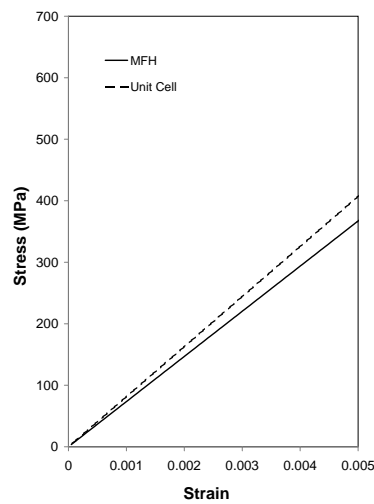

(b) $v_{\mathrm{I}}=0.30$

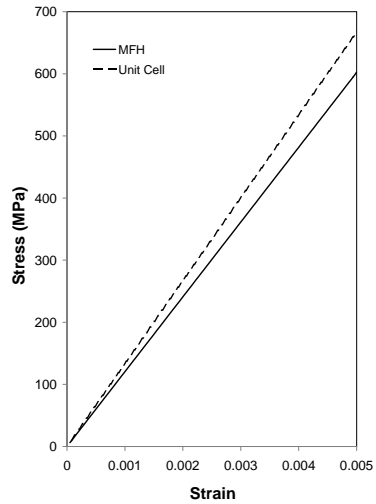

(c) $v_{\mathrm{I}}=0.50$

Figure 6: Stress-strain behavior of the epoxy fiber reinforced composite for different fiber volume ratios under longitudinal loading.

zone orthogonal to the direction of a crack propagation has been seen in the numerical simulations involving damage to fracture transition with constant $l$ $[49,61]$. Therefore, a variable length parameter should be considered to avoid this spurious behavior [49,61].

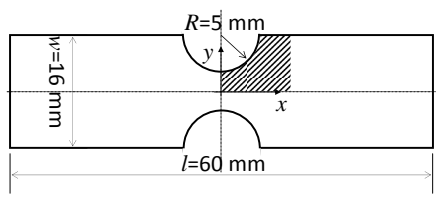

Figure 7: Schematics of the sample test.

The specimen, whose geometry is visualized in Fig. 7, represents the transverse section of a unidirectional reinforced ply. In order to study the effect of the non-local damage model, notches are added to force strain localization. The fibers are assumed to be perpendicular to this section. Thus plane-strain conditions are applied in the fiber direction (z-direction), and traction-free conditions are applied along the ply-thickness (y-direction). The specimen is then subjected to a transverse loading (x-direction), up to the softening stage. The material properties are the same as in the previous example with a fiber volume fraction of $v_{\mathrm{I}}=30 \%$. The symmetry of the specimen permits to model only one quarter of the structure in the finite element simulations.

The mesh of the quarter specimen is refined in the weak zone of $8.0 \times 8.0 \mathrm{~mm}$ (see the shadow area in Fig. 7), and three meshes are successively considered:

- Coarsest mesh of 182 elements and with a mesh size of about $0.43 \mathrm{~mm}$ at the notch ; 
- Intermediate mesh of 360 elements and with a mesh size of about $0.3 \mathrm{~mm}$ at the notch;

- Finest mesh of 1120 elements and with a the mesh size of about $0.15 \mathrm{~mm}$ at the notch.

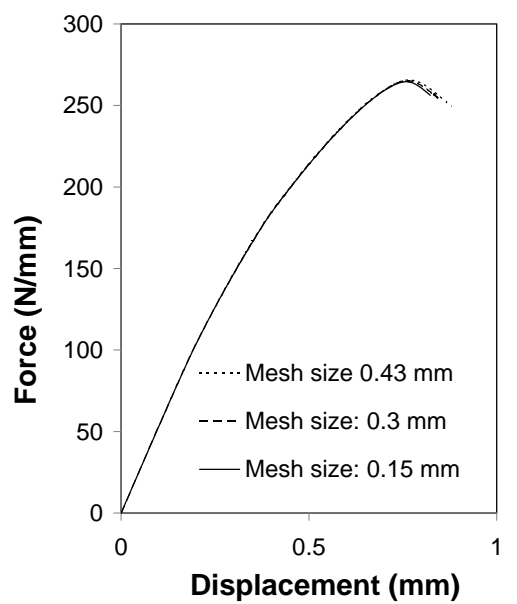

Figure 8: Force per unit ply length vs. displacement of the sample for different meshes.

The load-displacement curves are extracted for the three mesh sizes and are presented in Fig. 8. A good convergence can be seen with the decrease of the mesh size. Indeed, dividing the mesh size by 2 between the finest mesh and the intermediate mesh does not induce differences larger than between the coarsest and intermediate meshes, for which the mesh size has only been reduced by 30 $\%$.

For all the meshes, the computations did not converge further than $15 \%$ softening. This might be explained by the evolution of the damage in the matrix which obeys the Lemaitre-Chaboche model. Indeed, this model is characterized by a low damage increase rate at early loading stage while this rate increases abruptly at a given stage. For the studied example, the damage at the maximum displacement has already reached 0.25 , see Fig. 9(c) and a rather small increment of displacement would result in a damage reaching 1.0 at the stress concentration area. At this stage, a failure criterion could be derived, or a method for transition from damage to crack could be developed to pursue the simulation.

The basic characteristic of the gradient enhanced method resides in the existence of the non-local effect in the damage area: the evolution of the damage is not localized to a single element nor to a mesh dependent one-element-wide band. The interactions among elements are considered through the gradient items within a zone which size does not depend on the size of elements, but on the characteristic length. This phenomenon can be confirmed by the compu- 


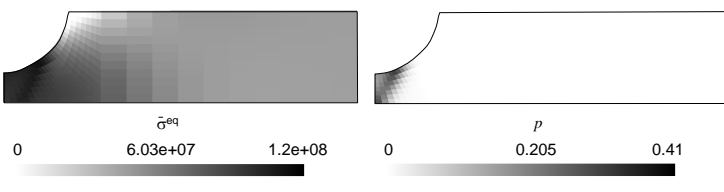

(a) Von Mises stress (Pa)

(b) Local accumulated plastic strain

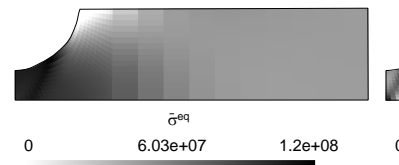

(d) Von Mises stress $(\mathrm{Pa})$

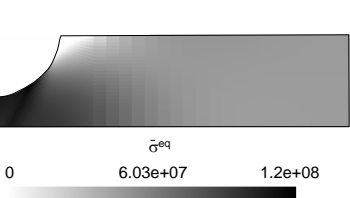

(g) Von Mises stress $(\mathrm{Pa})$

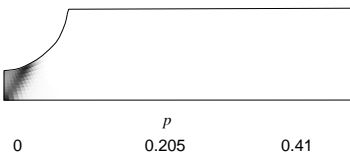

(e) Local accumulated plastic strain

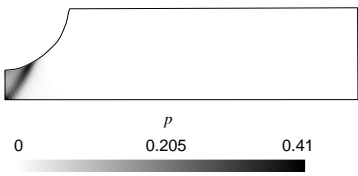

(h) Local accumulated plastic strain

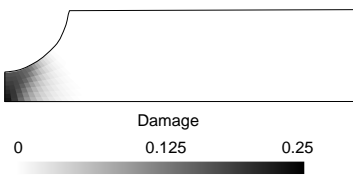

(c) Damage

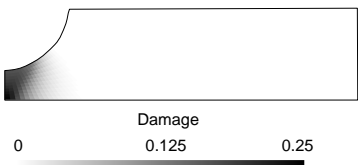

(f) Damage

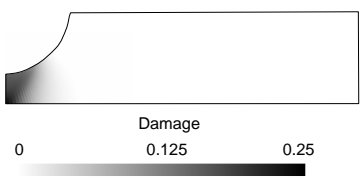

(i) Damage

Figure 9: The von Mises stress in the homogenized material, accumulated plastic strain in the matrix phase and damage in the matrix phase at maximum displacement, for coarsest mesh mesh size $=0.43 \mathrm{~mm}(\mathrm{a}) \sim(\mathrm{c})$, intermediate mesh - mesh size $=0.3 \mathrm{~mm}(\mathrm{~d}) \sim(\mathrm{f})$ and finest mesh - mesh size $=0.15 \mathrm{~mm}(\mathrm{~g}) \sim(\mathrm{i})$.

tational results. For the three different mesh cases, Fig. 9 displays the distributions of von Mises stress, accumulated plastic strain in matrix and damage in matrix at maximum displacement (about $0.83 \mathrm{~mm}$ ) respectively. These figures demonstrate the convergence of the method with respect to the mesh-size: for three different meshes, only small differences can be observed in the field distributions. From Fig. 9 it can be seen that the local accumulated plastic strain distributions form a band while the non-local damage distributions have a more diffuse distribution. However, from the accumulated plastic strain and damage distributions shown in Fig. 9, it can be seen that if the method is used to estimate a crack initiation, fine meshes are still required.

\section{Conclusions}

A gradient enhanced mean-field homogenization (MFH) scheme, based on the so-called linear comparison composite (LCC) approach, for damage-enhanced elasto-plastic composites, was presented.

In particular, at the micro-level, the fibers were assumed to remain elastic, while the matrix obeys an elasto-plastic behavior with damage. In order of avoiding issues related to softening behaviors, such as mesh-dependency, arbitrary strain localization ... the damage of the matrix was formulated in a 
non-local way, using the so-called implicit approach. In this approach, the damage is computed from a non-local equivalent accumulated plastic strain, which is obtained by solving new Helmholtz-type equations relating the non-local accumulated plastic strain to its local expression.

At the homogenized macro-level, the resulting formulation required four degrees of freedom per nodes, the three usual displacement unknowns, and a new degree of freedom, which is a representation of the equivalent accumulated plastic strain in the matrix. Thus, the set of traditional mechanical equations was completed in a fully coupled way by a new set of equations corresponding to the weak formulation of the non-local implicit formulation.

Although general, this approach was applied to unidirectional carbon-fiber, epoxy-matrix composites. First the responses under uniform strain obtained with the new non-local MFH formulation were compared to the responses obtained with the direct numerical simulation of periodical cells and RVEs. Toward this end, a loading/unloading cycle was applied in the transverse direction. For low fiber volume ratios $(<30 \%)$ results are in excellent agreement, including during the softening and unloading parts of the loading history. For average fiber volume ratios (up to $50 \%$ ), the method is less accurate (error up to $20 \%$ ), which is mainly explained by the M-T assumption and the incremental $\mathrm{MFH}$ approach adopted in this paper. Indeed MFH using the LCC incremental formulation does not correct the previous stress increments from a modification of the material stiffness, due to the plastic or damage behavior.

Finally the methodology was applied to study the transverse loading of a notched composite ply. In particular it was shown that the method can capture the softening and lead to results that converge with mesh refinement, demonstrating the ability of the method to be used to compute mesoscopic or macroscopic structures.

Nevertheless, the method was found to remain accurate for moderate fiber volume ratios only. The MFH could thus be enriched by second statistical moments [18] to improve the accuracy. Adding anisotropic behaviors in the fiber and/or in the damage law and in the characteristic length, as well as considering fiber debonding would allow to compare predictions with experimental results as more physical inputs would be accounted for. Also, in a future work the model will be used to predict macro-crack initiation, including comparisons to experimental results.

\section{Appendix A. Tensorial operations and notations}

- Dots and colons are used to indicate tensor products contracted over one and two indices respectively:

$$
\begin{aligned}
\boldsymbol{u} \cdot \boldsymbol{v}=u_{i} v_{i}, & (\boldsymbol{a} \cdot \boldsymbol{u})_{i}=a_{i j} u_{j} \\
(\boldsymbol{a} \cdot \boldsymbol{b})_{i j}=a_{i k} b_{k j}, & \boldsymbol{a}: \boldsymbol{b}=a_{i j} b_{j i} \\
(\boldsymbol{C}: \boldsymbol{a})_{i j}=C_{i j k l} a_{l k}, & (\boldsymbol{C}: \boldsymbol{D})_{i j k l}=C_{i j m n} D_{n m k l} .
\end{aligned}
$$


- Dyadic products are designated by $\otimes$ :

$$
(\boldsymbol{u} \otimes \boldsymbol{v})_{i j}=u_{i} v_{j}, \quad(\boldsymbol{a} \otimes \boldsymbol{b})_{i j k l}=a_{i j} b_{k l} .
$$

- Symbols 1 and $\boldsymbol{I}$ designate the second- and fourth-order symmetric identity tensors respectively:

$$
\mathbf{1}_{i j}=\delta_{i j}, \quad \boldsymbol{I}_{i j k l}=\frac{1}{2}\left(\delta_{i k} \delta_{j l}+\delta_{i l} \delta_{j k}\right),
$$

where $\delta_{i j}=1$ if $i=j, \delta_{i j}=0$ if $i \neq j$.

- The spherical and deviatoric operators are $\boldsymbol{I}^{\mathrm{vol}}$ and $\boldsymbol{I}^{\mathrm{dev}}$ respectively:

$$
\boldsymbol{I}^{\mathrm{vol}} \equiv \frac{1}{3} \mathbf{1} \otimes \mathbf{1}, \quad \boldsymbol{I}^{\mathrm{dev}}=\boldsymbol{I}-\boldsymbol{I}^{\mathrm{vol}},
$$

so that for symmetric tensors $a_{i j}=a_{j i}$ we have:

$$
\boldsymbol{I}^{\mathrm{vol}}: \boldsymbol{a}=\frac{1}{3} a_{m m} \mathbf{1}, \quad \boldsymbol{I}^{\mathrm{dev}}: \boldsymbol{a}=\boldsymbol{a}-\frac{1}{3} a_{m m} \mathbf{1}=\operatorname{dev}(\boldsymbol{a}) .
$$

- Any fourth-order tensors $\boldsymbol{C}$ and $\boldsymbol{D}$ define the following scalar invariant:

$$
\boldsymbol{C}:: \boldsymbol{D}=C_{i j k l} D_{l k j i}=\boldsymbol{D}:: \boldsymbol{C}
$$

Using this definition, the following results are easily obtained:

$$
\boldsymbol{I}^{\mathrm{vol}}:: \boldsymbol{I}^{\mathrm{vol}}=1, \quad \boldsymbol{I}^{\mathrm{dev}}:: \boldsymbol{I}^{\mathrm{dev}}=5 \quad \text { and } \quad \boldsymbol{I}^{\mathrm{vol}}:: \boldsymbol{I}^{\mathrm{dev}}=0 .
$$

- Any symmetric and isotropic fourth-order tensor $\boldsymbol{C}_{\text {iso }}$ is a linear combination of $\boldsymbol{I}^{\mathrm{vol}}$ and $\boldsymbol{I}^{\mathrm{dev}}$, and can be written as follows

$$
\boldsymbol{C}_{\text {iso }}=\left(\boldsymbol{I}^{\mathrm{vol}}:: \boldsymbol{C}^{\mathrm{iso}}\right) \boldsymbol{I}^{\mathrm{vol}}+\frac{1}{5}\left(\boldsymbol{I}^{\mathrm{dev}}:: \boldsymbol{C}^{\mathrm{iso}}\right) \boldsymbol{I}^{\mathrm{dev}}
$$

\section{Appendix B. The Lemaitre-Chaboche ductile damage model in non- local form}

In this section, subscript 0 related to the matrix material is omitted for simplicity.

In the "time-continuum" formulation of sec. 2.2.4, a "continuum" elastoplastic tangent operator such that $\dot{\hat{\sigma}}=C^{\mathrm{ep}}: \dot{\varepsilon}$ can be calculated from (e.g. [54, chapter 12]):

$$
\boldsymbol{C}^{\mathrm{ep}}=\boldsymbol{C}^{\mathrm{el}}-\frac{(2 \mu)^{2}}{h} \boldsymbol{N} \otimes \boldsymbol{N}, \quad h=3 \mu+\frac{\mathrm{d} R}{\mathrm{~d} p}>0,
$$

where $\mu$ is the shear modulus. Due to the finite increments of strain and stress between time $t_{n}$ and time $t_{n+1}$, the operator $\boldsymbol{C}^{\text {alg }}=\frac{\partial \Delta \hat{\boldsymbol{\sigma}}}{\partial \Delta \varepsilon}$ differs from $\boldsymbol{C}^{\mathrm{ep}}$, 
[50]. In the case of the radial return mapping assumption, the derivative of the effective stress increment with respect to the strain increment reads (e.g. [54, chapter 12])

$$
\boldsymbol{C}^{\mathrm{alg}}=\boldsymbol{C}^{\mathrm{ep}}-(2 \mu)^{2}(\Delta p) \frac{\hat{\sigma}^{\mathrm{eq}}}{\hat{\sigma}^{\mathrm{eq}, \operatorname{tr}}} \frac{\partial \boldsymbol{N}}{\partial \hat{\boldsymbol{\sigma}}}, \quad \text { with } \frac{\partial \boldsymbol{N}}{\partial \hat{\boldsymbol{\sigma}}}=\frac{1}{\hat{\sigma}^{\mathrm{eq}}}\left(\frac{3}{2} \boldsymbol{I}^{\mathrm{dev}}-\boldsymbol{N} \otimes \boldsymbol{N}\right) .
$$

In this last relation, $\hat{\sigma}^{\text {eq, tr }}$ is the trial (elastic predictor) value of $\hat{\sigma}_{\text {eq }}$, and $\Delta p$ is the accumulated plastic strain increment in the time interval. Note that in case of plastic flow both $\boldsymbol{C}^{\text {ep }}$ and $\boldsymbol{C}^{\text {alg }}$ are anisotropic, and that in case of elasticity $\boldsymbol{C}^{\text {alg }}$ reduces to $\boldsymbol{C}^{\mathrm{el}}$.

The increment of damage in one time step is expressed as [46]:

$$
\Delta D=\left(\frac{Y_{n+\alpha}}{S_{0}}\right)^{s} \Delta \tilde{p}
$$

where

$$
Y=\frac{1}{2} \varepsilon^{\mathrm{e}}: \boldsymbol{C}_{0}^{\mathrm{el}}: \boldsymbol{\varepsilon}^{\mathrm{e}} \quad \text { and } \quad Y_{n+\alpha}=(1-\alpha) Y_{n}+\alpha Y_{n+1}
$$

It can be easily deduced that

$$
\frac{\partial Y}{\partial \varepsilon^{\mathrm{e}}}: \frac{\partial \varepsilon^{\mathrm{e}}}{\partial \varepsilon}: \delta \varepsilon=\alpha \varepsilon^{\mathrm{e}}: C^{\mathrm{alg}}: \delta \varepsilon
$$

leading to

$$
\begin{aligned}
\delta D(\varepsilon, \tilde{p}) & \approx \frac{\partial \Delta D}{\partial Y} \frac{\partial Y}{\partial \varepsilon^{\mathrm{e}}}: \frac{\partial \varepsilon^{\mathrm{e}}}{\partial \varepsilon}: \delta \varepsilon+\frac{\partial \Delta D}{\partial \tilde{p}} \delta \tilde{p} \\
& =s \Delta \tilde{p} \frac{\left(Y_{n+\alpha}\right)^{s-1}}{S_{0}^{s}} \frac{\partial Y}{\partial \varepsilon^{\mathrm{e}}}: \frac{\partial \varepsilon^{\mathrm{e}}}{\partial \varepsilon}: \delta \varepsilon+\left(\frac{Y_{n+\alpha}}{S_{0}}\right)^{s} \delta \tilde{p} \\
& =\alpha s \Delta \tilde{p} \frac{\left(Y_{n+\alpha}\right)^{s-1}}{S_{0}^{s}} \varepsilon^{\mathrm{e}}: C^{\mathrm{alg}}: \delta \varepsilon+\left(\frac{Y_{n+\alpha}}{S_{0}}\right)^{s} \delta \tilde{p}
\end{aligned}
$$

\section{Appendix C. Stress residual vector}

The equation to be satisfied at the end of the MFH procedure is Eq. (30). Multiplying Eq. (28) by $\boldsymbol{B}^{\epsilon}\left(\boldsymbol{I}, \overline{\boldsymbol{C}}_{0}^{\text {algD }}{ }_{n+\alpha}, \overline{\boldsymbol{C}}_{\mathrm{I}}^{\text {alg }}{ }_{n+\alpha}\right)$ ans using (30) lead to

$$
\begin{array}{r}
v_{0} \Delta \boldsymbol{\varepsilon}_{\mathrm{I} n+1}+v_{\mathrm{I}} \boldsymbol{B}^{\epsilon}\left(\boldsymbol{I}, \overline{\boldsymbol{C}}_{0}^{\mathrm{alg}{ }_{n+\alpha},}, \overline{\boldsymbol{C}}_{\mathrm{I}}^{\mathrm{alg}}{ }_{n+\alpha}\right): \Delta \boldsymbol{\varepsilon}_{\mathrm{I} n+1}= \\
\boldsymbol{B}^{\epsilon}\left(\boldsymbol{I}, \overline{\boldsymbol{C}}_{0}^{\mathrm{algD}}{ }_{n+\alpha}, \overline{\boldsymbol{C}}_{\mathrm{I}}^{\mathrm{alg}}{ }_{n+\alpha}\right): \Delta \overline{\boldsymbol{\varepsilon}}_{n+1} .
\end{array}
$$

With the M-T assumption the strain concentration tensor follows from (4), and Eq. (C.1) reads

$$
\Delta \boldsymbol{\varepsilon}_{\mathrm{I} n+1}+v_{0} \boldsymbol{S}:\left[\left(\overline{\boldsymbol{C}}_{0}^{\mathrm{algD}}{ }_{n+\alpha}\right)^{-1}: \overline{\boldsymbol{C}}_{\mathrm{I}}^{\mathrm{alg}}{ }_{n+\alpha}-\boldsymbol{I}\right]: \Delta \boldsymbol{\varepsilon}_{\mathrm{I} n+1}=\Delta \overline{\boldsymbol{\varepsilon}}_{n+1},
$$


or again $\boldsymbol{F}=0$ with

$$
\begin{aligned}
\boldsymbol{F}= & \overline{\boldsymbol{C}}_{0}^{\mathrm{algD}}{ }_{n+\alpha}:\left[\Delta \varepsilon_{\mathrm{I} n+1}-\frac{1}{v_{0}} \boldsymbol{S}^{-1}:\left(\Delta \varepsilon_{\mathrm{I} n+1}-\Delta \overline{\boldsymbol{\varepsilon}}_{n+1}\right)\right] \\
& -\overline{\boldsymbol{C}}_{\mathrm{I}}^{\mathrm{alg}}{ }_{n+\alpha}: \Delta \boldsymbol{\varepsilon}_{\mathrm{I} n+1} .
\end{aligned}
$$

In order to satisfy $\boldsymbol{F}=0$, Eq. (C.3) is linearized as

$$
\mathrm{d} \boldsymbol{F}=\frac{\partial \boldsymbol{F}}{\partial \varepsilon_{\mathrm{I}}}: \mathrm{d} \Delta \varepsilon_{\mathrm{I}}+\frac{\partial \boldsymbol{F}}{\partial \varepsilon_{0}}: \mathrm{d} \Delta \varepsilon_{0}+\frac{\partial \boldsymbol{F}}{\Delta \bar{\varepsilon}}: \mathrm{d} \Delta \overline{\boldsymbol{\varepsilon}}+\frac{\partial \boldsymbol{F}}{\partial \tilde{p}} \mathrm{~d} \tilde{p} .
$$

When solving $\boldsymbol{F}=0$ at constant $\Delta \bar{\varepsilon}$ and constant $\tilde{p}$, as $v_{0} \Delta \varepsilon_{0}+v_{\mathrm{I}} \Delta \varepsilon_{\mathrm{I}}$ is also constant, the iteration process relies on $\mathrm{d} \boldsymbol{F}=\boldsymbol{J}$ : $\mathrm{d} \boldsymbol{\varepsilon}_{\mathrm{I}}$ with

$$
\begin{aligned}
\boldsymbol{J}= & \frac{\partial \boldsymbol{F}}{\partial \boldsymbol{\varepsilon}_{\mathrm{I}}}+\frac{\partial \boldsymbol{F}}{\partial \boldsymbol{\varepsilon}_{0}}: \frac{\partial \boldsymbol{\varepsilon}_{0}}{\partial \boldsymbol{\varepsilon}_{\mathrm{I}}} \\
= & \overline{\boldsymbol{C}}_{0}^{\mathrm{algD}}{ }_{n+\alpha}:\left[\boldsymbol{I}-\boldsymbol{S}^{-1}\right]-\overline{\boldsymbol{C}}_{\mathrm{I}}^{\mathrm{alg}}{ }_{n+\alpha}-\frac{\partial \overline{\boldsymbol{C}}_{\mathrm{I}}^{\mathrm{alg}}{ }_{n+\alpha}}{\partial \boldsymbol{\varepsilon}_{\mathrm{I}}}: \Delta \boldsymbol{\varepsilon}_{\mathrm{I} n+1}- \\
& \frac{v_{\mathrm{I}}}{v_{0}} \frac{\partial \overline{\boldsymbol{C}}_{0}^{\mathrm{algD}}{ }_{n+\alpha}}{\partial \boldsymbol{\varepsilon}_{0}}:\left[\Delta \boldsymbol{\varepsilon}_{\mathrm{I} n+1}-\boldsymbol{S}^{-1}: \frac{\left(\Delta \boldsymbol{\varepsilon}_{\mathrm{I} n+1}-\Delta \overline{\boldsymbol{\varepsilon}}_{n+1}\right)}{v_{0}}\right]- \\
& \frac{v_{\mathrm{I}}}{v_{0}} \overline{\boldsymbol{C}}_{0}^{\mathrm{algD}}{ }_{n+\alpha}: \boldsymbol{S}^{-1}
\end{aligned}
$$

Once $\boldsymbol{F}=0$ is satisfied, the effect on the strain increment in each phase of a variation $\mathrm{d} \Delta \bar{\varepsilon}$ at constant $\Delta \tilde{p}$ can directly be obtained by constraining $\mathrm{d} \boldsymbol{F}=0$, and Eq. (C.4) leads to

$$
0=\frac{\partial \boldsymbol{F}}{\partial \boldsymbol{\varepsilon}_{\mathrm{I}}}: \mathrm{d} \Delta \varepsilon_{\mathrm{I}}+\frac{\partial \boldsymbol{F}}{\partial \varepsilon_{0}}: \mathrm{d} \Delta \varepsilon_{0}+\frac{\partial \boldsymbol{F}}{\partial \bar{\varepsilon}}: \mathrm{d} \Delta \bar{\varepsilon},
$$

or again

$$
\frac{\partial \varepsilon_{\mathrm{I}}}{\partial \bar{\varepsilon}}=-\boldsymbol{J}^{-1}: \frac{\partial \boldsymbol{F}}{\partial \bar{\varepsilon}} .
$$

As under these circumstances $\mathrm{d} \overline{\boldsymbol{\varepsilon}}=v_{0} \mathrm{~d} \varepsilon_{0}+v_{\mathrm{I}} \mathrm{d} \boldsymbol{\varepsilon}_{\mathrm{I}}$, this last equation is completed by

$$
\frac{\partial \varepsilon_{0}}{\partial \bar{\varepsilon}}=\frac{1}{v_{0}}\left(\boldsymbol{I}-v_{1} \frac{\partial \varepsilon_{\mathrm{I}}}{\partial \bar{\varepsilon}}\right) .
$$

The same relations can be obtained for a linearization with respect to $\tilde{p}$ at constant $\Delta \bar{\varepsilon}$.

\section{Acknowledgment}

The research has been funded by the Walloon Region under the agreement SIMUCOMP $n^{\circ} 1017232$ (CT-EUC 2010-10-12) in the context of the ERA-NET + , Matera + framework. 


\section{References}

[1] P. Kanouté, D. Boso, J. Chaboche, B. Schrefler, Multiscale Methods for Composites: A Review, Archives of Computational Methods in Engineering 16 (2009) 31-75, ISSN 1134-3060, 10.1007/s11831-008-9028-8.

[2] J. D. Eshelby, The Determination of the Elastic Field of an Ellipsoidal Inclusion, and Related Problems, Proceedings of the Royal Society of London. Series A, Mathematical and Physical Sciences 241 (1226) (1957) pp. 376-396, ISSN 00804630.

[3] T. Mori, K. Tanaka, Average stress in matrix and average elastic energy of materials with misfitting inclusions, Acta Metallurgica 21 (5) (1973) 571-574, cited By (since 1996) 1814.

[4] Y. Benveniste, A new approach to the application of Mori-Tanaka's theory in composite materials, Mechanics of Materials 6 (2) (1987) $147-157$, ISSN 0167-6636, doi:DOI: 10.1016/0167-6636(87)90005-6.

[5] B. Budiansky, On the elastic moduli of some heterogeneous materials, Journal of the Mechanics and Physics of Solids 13 (4) (1965) 223 - 227, ISSN 0022-5096, doi:DOI: 10.1016/0022-5096(65)90011-6.

[6] E. Kröner, Berechnung der elastischen Konstanten des Vielkristalls aus den Konstanten des Einkristalls, Zeitschrift für Physik A Hadrons and Nuclei 151 (1958) 504-518, ISSN 0939-7922, 10.1007/BF01337948.

[7] A. Hershey, The elasticity of an isotropic aggregate of anisotropic cubic crystals, Journal of Applied mechanics-transactions of the ASME 21 (3) (1954) 236-240.

[8] R. Hill, A self-consistent mechanics of composite materials, Journal of the Mechanics and Physics of Solids 13 (4) (1965) 213 - 222, ISSN 0022-5096, doi:DOI: 10.1016/0022-5096(65)90010-4.

[9] D. R. S. Talbot, J. R. Willis, Variational Principles for Inhomogeneous Nonlinear Media, IMA Journal of Applied Mathematics 35 (1) (1985) 39-54, doi:10.1093/imamat/35.1.39.

[10] D. R. S. Talbot, J. R. Willis, Bounds and Self-Consistent Estimates for the Overall Properties of Nonlinear Composites, IMA Journal of Applied Mathematics 39 (3) (1987) 215-240, doi:10.1093/imamat/39.3.215.

[11] P. Ponte Castañeda, The effective mechanical properties of nonlinear isotropic composites, Journal of the Mechanics and Physics of Solids 39 (1) (1991) 45-71, ISSN 0022-5096, doi:DOI: 10.1016/0022-5096(91)90030-R.

[12] P. Ponte Castañeda, A New Variational Principle and its Application to Nonlinear Heterogeneous Systems, SIAM Journal on Applied Mathematics 52 (5) (1992) 1321-1341, ISSN 00361399. 
[13] D. Talbot, J. Willis, Some simple explicit bounds for the overall behaviour of nonlinear composites, International Journal of Solids and Structures 29 (14-15) (1992) 1981 - 1987, ISSN 0020-7683, doi:DOI: 10.1016/00207683(92)90188-Y.

[14] R. Hill, Continuum micro-mechanics of elastoplastic polycrystals, Journal of the Mechanics and Physics of Solids 13 (2) (1965) 89 - 101, ISSN 00225096, doi:DOI: 10.1016/0022-5096(65)90023-2.

[15] H. E. Pettermann, A. F. Plankensteiner, H. J. Böhm, F. G. Rammerstorfer, A thermo-elasto-plastic constitutive law for inhomogeneous materials based on an incremental Mori-Tanaka approach, Computers \& Structures 71 (2) (1999) 197 - 214, ISSN 0045-7949, doi:DOI: 10.1016/S0045-7949(98)002089.

[16] I. Doghri, A. Ouaar, Homogenization of two-phase elasto-plastic composite materials and structures: Study of tangent operators, cyclic plasticity and numerical algorithms, International Journal of Solids and Structures 40 (7) (2003) 1681 - 1712, ISSN 0020-7683, doi:DOI: 10.1016/S00207683(03)00013-1.

[17] J. Chaboche, P. Kanouté, A. Roos, On the capabilities of mean-field approaches for the description of plasticity in metal matrix composites, International Journal of Plasticity 21 (7) (2005) 1409 - 1434, ISSN 0749-6419, doi:DOI: 10.1016/j.ijplas.2004.07.001.

[18] I. Doghri, L. Brassart, L. Adam, J. S. Gérard, A second-moment incremental formulation for the mean-field homogenization of elasto-plastic composites, International Journal of Plasticity 27 (3) (2011) 352 - 371, ISSN 0749-6419, doi:DOI: 10.1016/j.ijplas.2010.06.004.

[19] V. Kouznetsova, M. G. D. Geers, W. A. M. Brekelmans, Multi-scale constitutive modelling of heterogeneous materials with a gradient-enhanced computational homogenization scheme, International Journal for Numerical Methods in Engineering 54 (8) (2002) 1235-1260.

[20] V. Kouznetsova, M. Geers, W. Brekelmans, Multi-scale second-order computational homogenization of multi-phase materials: a nested finite element solution strategy, Computer Methods in Applied Mechanics and Engineering 193 (48-51) (2004) 5525 - 5550, ISSN 0045-7825, doi:DOI: 10.1016/j.cma.2003.12.073, advances in Computational Plasticity.

[21] R. De Borst, Simulation of strain localization: a reappraisal of the Cosserat continuum, Engineering Computations 8 (4) (1991) 317 - 332, ISSN 02644401.

[22] Z. P. Bazant, T. B. Belytchko, T. P. Chang, Continuum Theory for StrainSoftening, Journal of Engineering Mechanics-ASCE 110 (12) (1984) 16661692, doi:10.1061/(ASCE)0733-9399(1984)110:12(1666). 
[23] H. M. Zbib, E. C. Aifantis, A Gradient-Dependent Flow Theory of Plasticity: Application to Metal and Soil Instabilities, Applied Mechanics Reviews 42 (11S) (1989) S295-S304, doi:10.1115/1.3152403.

[24] R. De Borst, L. Sluys, H.-B. Mühlhaus, J. Pamin, Fundamental issues in finite element analyses of localization of deformation, Engineering Computations 10 (2) (1993) 99 - 121, ISSN 0264-4401.

[25] M. Geers, Experimental Analysis and Computational Modelling of Damage and Fracture, Ph.D. thesis, University of Technology, Eindhoven (Netherlands)., 1997.

[26] R. Peerlings, R. de Borst, W. Brekelmans, M. Geers, Gradient-enhanced damage modelling of concrete fracture, Mech. Cohesive-Frictional Mat. 3 (1998) 323-342.

[27] R. Peerlings, R. de Borst, W. Brekelmans, J. de Vree, Gradient-enhanced damage for quasi-brittle materials, Int. J. Numer. Meth. Engng 39 (1996) 3391-3403.

[28] X. Liu, G. Hu, A continuum micromechanical theory of overall plasticity for particulate composites including particle size effect, International Journal of Plasticity 21 (4) (2005) 777 - 799, ISSN 0749-6419, doi: 10.1016/j.ijplas.2004.04.014.

[29] C. Dascalu, A two-scale damage model with material length, Comptes Rendus Mécanique 337 (9-10) (2009) 645 - 652, ISSN 1631-0721, doi: 10.1016/j.crme.2009.09.008.

[30] R. Knockaert, I. Doghri, Nonlocal constitutive models with gradients of internal variables derived from a micro/macro homogenization procedure, Computer Methods in Applied Mechanics and Engineering 174 (1-2) (1999) 121 - 136, ISSN 0045-7825, doi:DOI: 10.1016/S0045-7825(98)00282-5.

[31] T. Massart, R. Peerlings, M. Geers, A dissipation-based control method for the multi-scale modelling of quasi-brittle materials, C. R. Mecanique 333 (2005) 521527.

[32] T. Massart, R. Peerlings, M. Geers, An enhanced multi-scale approach for masonry wall computations, Int. J. Numer. Meth. Engng 69 (5) (2007) $1022-1059$.

[33] E. Coenen, V. Kouznetsova, M. Geers, Enabling microstructure-based damage and localization analyses and upscaling, Modelling and Simulation in Materials Science and Engineering 19 (7) (2011) 074008.

[34] E. Coenen, V. Kouznetsova, M. Geers, Novel boundary conditions for strain localization analyses in microstructural volume elements, International Journal for Numerical Methods in Engineering . 
[35] R. Peerlings, M. Geers, R. de Borst, W. Brekelmans, A critical comparison of nonlocal and gradient-enhanced softening continua, Int. J. Solids Structures 38 (2001) 7723-7746.

[36] J. Segurado, J. Llorca, A numerical approximation to the elastic properties of sphere-reinforced composites, Journal of the Mechanics and Physics of Solids 50 (10) (2002) 2107 - 2121, ISSN 0022-5096.

[37] M. Berveiller, A. Zaoui, An extension of the self-consistent scheme to plastically-flowing polycrystals, Journal of the Mechanics and Physics of Solids 26 (5-6) (1978) 325 - 344, ISSN 0022-5096, doi:DOI: 10.1016/00225096(78)90003-0.

[38] G. J. Weng, The overall elastoplastic stress-strain relations of dual-phase metals, Journal of the Mechanics and Physics of Solids 38 (3) (1990) 419 441, ISSN 0022-5096, doi:DOI: 10.1016/0022-5096(90)90007-Q.

[39] P. Ponte Castañeda, Exact second-order estimates for the effective mechanical properties of nonlinear composite materials, Journal of the Mechanics and Physics of Solids 44 (6) (1996) 827 - 862, ISSN 0022-5096, doi:DOI: 10.1016/0022-5096(96)00015-4.

[40] L. Brassart, L. Stainier, I. Doghri, L. Delannay, A variational formulation for the incremental homogenization of elasto-plastic composites, Journal of the Mechanics and Physics of Solids 59 (12) (2011) 2455 - 2475, ISSN 0022-5096, doi:10.1016/j.jmps.2011.09.004.

[41] N. Lahellec, P. Suquet, On the effective behavior of nonlinear inelastic composites: I. Incremental variational principles, Journal of the Mechanics and Physics of Solids 55 (9) (2007) 1932 - 1963, ISSN 0022-5096, doi: 10.1016/j.jmps.2007.02.003.

[42] N. Lahellec, P. Ponte Castañeda, P. Suquet, Variational estimates for the effective response and field statistics in thermoelastic composites with intraphase property fluctuations, Proceedings of the Royal Society A: Mathematical, Physical and Engineering Science 467 (2132) (2011) 2224-2246, doi:10.1098/rspa.2010.0609.

[43] R. Masson, M. Bornert, P. Suquet, A. Zaoui, An affine formulation for the prediction of the effective properties of nonlinear composites and polycrystals, Journal of The Mechanics and Physics of Solids 48 (2000) 1203-1227, doi:10.1016/S0022-5096(99)00071-X.

[44] O. Pierard, I. Doghri, Study of Various Estimates of the Macroscopic Tangent Operator in the Incremental Homogenization of Elastoplastic Composites, International Journal for Multiscale Computational Engineering 4 (4) (2006) 521-543, ISSN 1543-1649. 
[45] Z. P. Bazant, G. Pijaudier-Cabot, Nonlocal Continuum Damage, Localization Instability and Convergence, Journal of Applied Mechanics 55 (2) (1988) 287-293, doi:10.1115/1.3173674, URL http://link. aip.org/link/?AMJ/55/287/1.

[46] J. Lemaitre, R. Desmorat, Engineering damage mechanics: ductile, creep, fatigue and brittle failures, Springer-Verlag, Berlin, ISBN 3540215034, 2005.

[47] J. Lemaitre, Coupled elasto-plasticity and damage constitutive equations, Computer Methods in Applied Mechanics and Engineering 51 (1-3) (1985) 31 - 49, ISSN 0045-7825, doi:DOI: 10.1016/0045-7825(85)90026-X.

[48] I. Doghri, Numerical implementation and analysis of a class of metal plasticity models coupled with ductile damage, International Journal for $\mathrm{Nu}-$ merical Methods in Engineering 38 (20) (1995) 3403-3431, ISSN 1097-0207, doi:10.1002/nme.1620382004.

[49] M. G. D. Geers, R. de Borst, W. A. M. Brekelmans, R. H. J. Peerlings, Strain-based transient-gradient damage model for failure analyses, Computer methods in applied mechanics and engineering 160 (1998) 133-153.

[50] J. C. Simo, R. L. Taylor, Consistent tangent operators for rate-independent elastoplasticity, Computer Methods in Applied Mechanics and Engineering 48 (1) (1985) 101 - 118, ISSN 0045-7825, doi:DOI: 10.1016/00457825(85)90070-2.

[51] G. J. Dvorak, Transformation Field Analysis of Inelastic Composite Materials, Proceedings: Mathematical and Physical Sciences 437 (1900) (1992) 311-327.

[52] G. J. Dvorak, Y. A. Bahei-El-Din, A. M. Wafa, Implementation of the transformation field analysis for inelastic composite materials, Computational Mechanics 14 (3) (1994) 201-228.

[53] L. Brassart, Homogenization of elasto-(visco)plastic composites: historydependent incremental and variational approaches, Ph.D. thesis, The Universit catholique de Louvain, Louvain-la-Neuve (Belgium)., 2011.

[54] I. Doghri, Mechanics of Deformable Solids- Linear, Nonlinear, Analytical and Computational Aspects, Springer-Verlag, Berlin, ISBN 3540669604, 2000 .

[55] R. Peerlings, T. Massart, M. Geers, A thermodynamically motivated implicit gradient damage framework and its application to brick masonry cracking, Computer Methods in Applied Mechanics and Engineering $193 \quad$ (30) (2004) $3403 \quad-$ 3417, ISSN 0045-7825, doi:10.1016/j.cma.2003.10.021, URL http://www.sciencedirect.com/science/article/pii/S0045782504001380. 
[56] C. Geuzaine, J.-F. Remacle, Gmsh: A 3-D finite element mesh generator with built-in pre- and post-processing facilities, International Journal for Numerical Methods in Engineering 79 (11) (2009) 1309-1331, URL http://dx.doi.org/10.1002/nme.2579.

[57] I. Gitman, H. Askes, L. Sluys, Representative volume: Existence and size determination, Engineering Fracture Mechanics 74 (16) (2007) 2518 - 2534, ISSN 0013-7944, doi:10.1016/j.engfracmech.2006.12.021, URL http://www.sciencedirect.com/science/article/pii/S0013794406004772.

[58] C. Pelissou, J. Baccou, Y. Monerie, F. Perales, Determination of the size of the representative volume element for random quasi-brittle composites, International Journal of Solids and Structures 46 (14-15) (2009) 2842 - 2855, ISSN 0020-7683, doi:10.1016/j.ijsolstr.2009.03.015, URL http://www.sciencedirect.com/science/article/pii/S0020768309001334.

[59] V. P. Nguyen, O. Lloberas-Valls, M. Stroeven, L. J. n. Sluys, On the existence of representative volumes for softening quasi-brittle materials - A failure zone averaging scheme, Computer Methods in Applied Mechanics and Engineering 199 (45-48) (2010) 3028 - 3038, ISSN 0045-7825, doi:10.1016/j.cma.2010.06.018, URL http://www.sciencedirect.com/science/article/pii/S0045782510001854.

[60] C. González, J. LLorca, Multiscale modeling of fracture in fiber-reinforced composites, Acta Materialia 54 (16) (2006) 4171 - 4181, ISSN 1359-6454.

[61] M. Geers, R. de Borst, W. Brekelmans, R. Peerlings, Validation and internal length scale determination for a gradient damage model: application to short glass-fibre-reinforced polypropylene, International Journal of Solids and Structures 36 (17) (1999) 2557 - 2583, ISSN 0020-7683. 